\title{
The Influence of Convective Momentum Transport and Vertical Wind Shear on the Evolution of a Cold Air Outbreak
}

\section{Journal Article}

Author(s):

Saggiorato, Beatrice; Nuijens, Louise; Siebesma, A. Pier; De Roode, Stephan R.; Sandu, Irina; Papritz, Lukas (D)

Publication date:

2020-06

Permanent link:

https://doi.org/10.3929/ethz-b-000424127

Rights / license:

Creative Commons Attribution 4.0 International

Originally published in:

Journal of Advances in Modeling Earth Systems 12(6), https://doi.org/10.1029/2019MS001991 


\section{JMES Journal of Advances in Modeling Earth Systems}

\author{
RESEARCH ARTICLE \\ 10.1029/2019MS001991 \\ Key Points: \\ - Momentum transport and its \\ contribution by convection can lead \\ to substantially different surface \\ winds under different wind shear \\ - Mesoscale circulations associated \\ with clouds can oppose turbulent \\ drag (accelerate winds) under \\ forward shear \\ - Wind shear can speed up the \\ stratocumulus-to-cumulus \\ transition by influencing surface \\ heat fluxes via momentum \\ transport
}

Correspondence to:

B. Saggiorato,

b.saggiorato@tudelft.nl

Citation:

Saggiorato, B., Nuijens, L., Siebesma, A. P., de Roode, S., Sandu, I., \& Papritz, L. (2020). The influence of convective momentum transport and vertical wind shear on the evolution of a cold air outbreak. Journal of Advances in Modeling Earth Systems, 12, e2019MS001991. https:// doi.org/10.1029/2019MS001991

Received 17 DEC 2019 Accepted 10 MAR 2020 Accepted article online 24 MAR 2020

(C)2020. The Authors.

This is an open access article under the terms of the Creative Commons Attribution License, which permits use, distribution and reproduction in any medium, provided the original work is properly cited.

\section{The Influence of Convective Momentum Transport and Vertical Wind Shear on the Evolution of a Cold Air Outbreak}

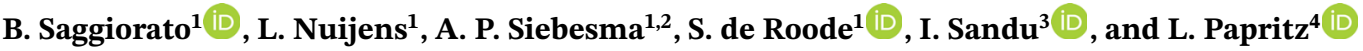 \\ ${ }^{1}$ Geoscience and Remote Sensing, Delft University of Technology, Delft, The Netherlands, ${ }^{2}$ Royal Netherlands \\ Meteorological Institute (KNMI), De Bilt, The Netherlands, ${ }^{3}$ European Centre for Medium-Range Weather Forecasts \\ (ECMWF), Reading, UK, ${ }^{4}$ Institute for Atmospheric and Climate Science, ETH, Zürich, Switzerland
}

\begin{abstract}
To study the influence of convective momentum transport (CMT) on wind, boundary layer and cloud evolution in a marine cold air outbreak (CAO) we use large-eddy simulations subject to different baroclinicity (wind shear) but similar surface forcing. The simulated domain is large enough, $\mathrm{O}\left(100 \times 100 \mathrm{~km}^{2}\right)$, to develop typical mesoscale cellular convective structures. We find that a maximum friction induced by momentum transport (MT) locates in the cloud layer for an increase of geostrophic wind with height (forward shear, FW) and near the surface for a decrease of wind with height (backward shear, BW). Although the total MT always acts as a friction, the interaction of friction-induced cross-isobaric flow with the Coriolis force can develop supergeostrophic winds near the surface (FW) or in the cloud layer (BW). The contribution of convection to MT is evaluated by decomposing the momentum flux by column water vapor and eddy size, revealing that CMT acts to accelerate subcloud layer winds under FW shear and that mesoscale circulations contribute significantly to MT for this horizontal resolution $(250 \mathrm{~m})$, even if small-scale eddies are nonnegligible and likely more important as resolution increases. Under FW shear, a deeper boundary layer and faster cloud transition are simulated, because MT acts to increase surface fluxes and wind shear enhances turbulent mixing across cloud tops. Our results show that the coupling between winds and convection is crucial for a range of problems, from CAO lifetime and cloud transitions to ocean heat loss and near-surface wind variability.
\end{abstract}

Plain Language Summary The vertical mixing of wind speed by shallow convection and clouds (called convective momentum transport, CMT) may play an important role in explaining boundary layer winds in midlatitude weather systems. In this study we use high-resolution simulations to study the influence of CMT on the evolution of winds and clouds in a typical high-latitude weather system: a cold air outbreak. In a cold air outbreak, strong surface fluxes and strong winds lead to extensive cloud decks that evolve as the system travels over increasingly warmer waters. To exemplify the role of wind mixing on surface winds and clouds we run simulations that are subject to different wind shear: from an increase of wind with height (Forward shear; FW) to a decrease of wind with height (backward shear; BW). We find that wind mixing always acts to slow down winds in the main flow direction, but the height where drag maximizes depends on the direction of shear. Whereas small-scale turbulence always acts as a drag, the mesoscale circulations and clouds themselves can speed up winds under FW shear. Enhanced turbulent mixing across cloud top and faster surface winds under FW shear also lead the clouds to evolve faster from closed-deck stratocumulus to broken cumulus fields, which is important for their radiative impact. Our results show that CMT has a significant influence on surface winds and is thus important for understanding air-sea interaction and near-surface wind variability, and as such, wind power generation.

\section{Introduction}

The influence of convective momentum transport (CMT) by shallow moist convection on large-scale atmospheric circulations is not well understood. One of the reasons is that studies of shallow convection have traditionally focused on first-order effects of shallow convection, such as vertical mixing of moisture and cloud formation, as well as their influence on the energy budget. Another reason is that the turbulence-resolving models, which we use to study shallow convection, are run on domain sizes much smaller than that of atmospheric weather systems, so that the large-scale wind is traditionally prescribed. 
As such, it has perhaps too long been interpreted as a forcing unchanged by convection itself. Yet recent sensitivity tests with the European Center of Medium-range Weather Forecasting (ECMWF) IFS model show that long-standing biases in near-surface wind speed and direction over global oceans (Sandu et al., 2013) may be linked to momentum transport by shallow convection. In this study, we wish to better understand the importance of CMT in the momentum budget of cloud-dominated atmospheres that are subject to a different baroclinicity, for example, vertical shear in the large-scale horizontal wind.

The system we focus on is a marine cold air outbreak (CAO), wherein a coupling between winds and convection seems particularly pronounced. In a CAO, polar or cold continental air masses are advected over relatively warm oceans, which trigger large sensible and latent heat fluxes at the air-sea interface (Grossman \& Betts, 1990; Papritz \& Spengler, 2017; Renfrew \& Moore, 1999; Wayland \& Raman, 1989). These large surface heat and moisture fluxes drive strong turbulence and convection, which deepen the boundary layer from several hundred meters to typically $2 \mathrm{~km}$ and more in a period of several hours (Brümmer, 1996; Fletcher et al., 2016a). Clouds are abundant and often have pronounced mesoscale features, such as open-cellular convection or cloud streets further downstream along a CAO trajectory (Brümmer, 1999). The large surface heat fluxes are driven not only by the pronounced air-sea temperature difference but also by stronger surface winds (Kolstad, 2017). While air-sea temperature differences diminish in response to large surface heat fluxes along CAO trajectories (Papritz et al., 2015), strong winds can help maintain those large fluxes. Strong surface winds, in turn, can be maintained by vertical mixing in the boundary layer, which can provide a downward flux of higher momentum air toward the surface, where winds are generally slowed due to surface drag. Climatologies of CAO's indeed reveal that low-level wind shear in the center of CAO's can reach values well above $5 \mathrm{~ms}^{-1} \cdot 100 \mathrm{hPa}^{-1}$ during the initial stage of a CAO (Fletcher et al., 2016a, 2016b). Typically, the shear becomes smaller throughout their evolution, which can be a signature of efficient vertical mixing. At the same time, CAO's tend to form in environments with pronounced vertical shear above the boundary layer, and more intense CAO's tend to form under somewhat stronger shear.

If CMT helps to maintain large surface heat fluxes, it is important not only for the lifetime of the CAO but also for the buoyancy flux forcing of the ocean mixed layer (Brümmer, 1996; Marshall \& Schott, 1999). Furthermore, the mesoscale organization of convection and clouds appears closely linked to the mesoscale variations in near-surface winds that are typically observed over oceans $(\mathcal{O}(2-100 \mathrm{~km})$ ) (Overland \& Wilson, 1984). Such large horizontal gradients in winds are important for marine activities and offshore wind energy production.

Yet representing the effects of characteristic mesoscale features in clouds and winds in a CAO is challenging for all global numerical models, including those that perform at "gray zone" resolutions, whereby convective or turbulent processes are partly resolved by the model and partly parameterized (De Roode et al., 2019; Tomassini et al., 2017). The Working Group on Numerical Experimentation (WGNE) GreyZone project used a CAO as their first case study to investigate whether parameterizations in global models can capture the observed cloudiness, boundary layer, and mesoscale structures. The GreyZone project emphasized that not just the scale-awareness of the convection scheme but also the interaction of the convection with the boundary layer scheme are important issues to be studied in order to improve model performance. The relative role of convection versus turbulence seems particularly important for the momentum flux. A handful of large eddy simulation (LES) studies have explored the nature of CMT and its representation through conventional mass flux schemes as used in global models (Brown, 1999; Kershaw \& Gregory, 1997; Siebesma et al., 2003; Schlemmer et al., 2017; Zhu, 2015). These studies reveal that a mass flux scheme may not fundamentally be appropriate for the momentum flux, which has significant contributions from clear-sky turbulence even in the cloud layer and is additionally altered by local pressure gradients and gravity waves. A recent study by Larson et al. (2019) shows that prognosing momentum fluxes with a higher-order model closed with an assumed probability density function works well for subtropical shallow cumulus cases in which the momentum flux has a three-layer structure, with downgradient momentum flux below cloud base, countergradient momentum flux through cloud base, and weak momentum flux in the cloud layer.

The objective of our study is to better understand how CMT helps set mean boundary layer winds in the midlatitudes, for example, can it explain the weak low-level wind shear as found in climatologies of CAOs. Second, we wish to understand the relative influence of turbulent mixing versus coherent updrafts or mesoscale circulations (the convection) to the momentum flux. And finally, we question how the cloud and boundary layer transitions that are so typical of a CAO depend on vertical shear (via the effect of CMT). 

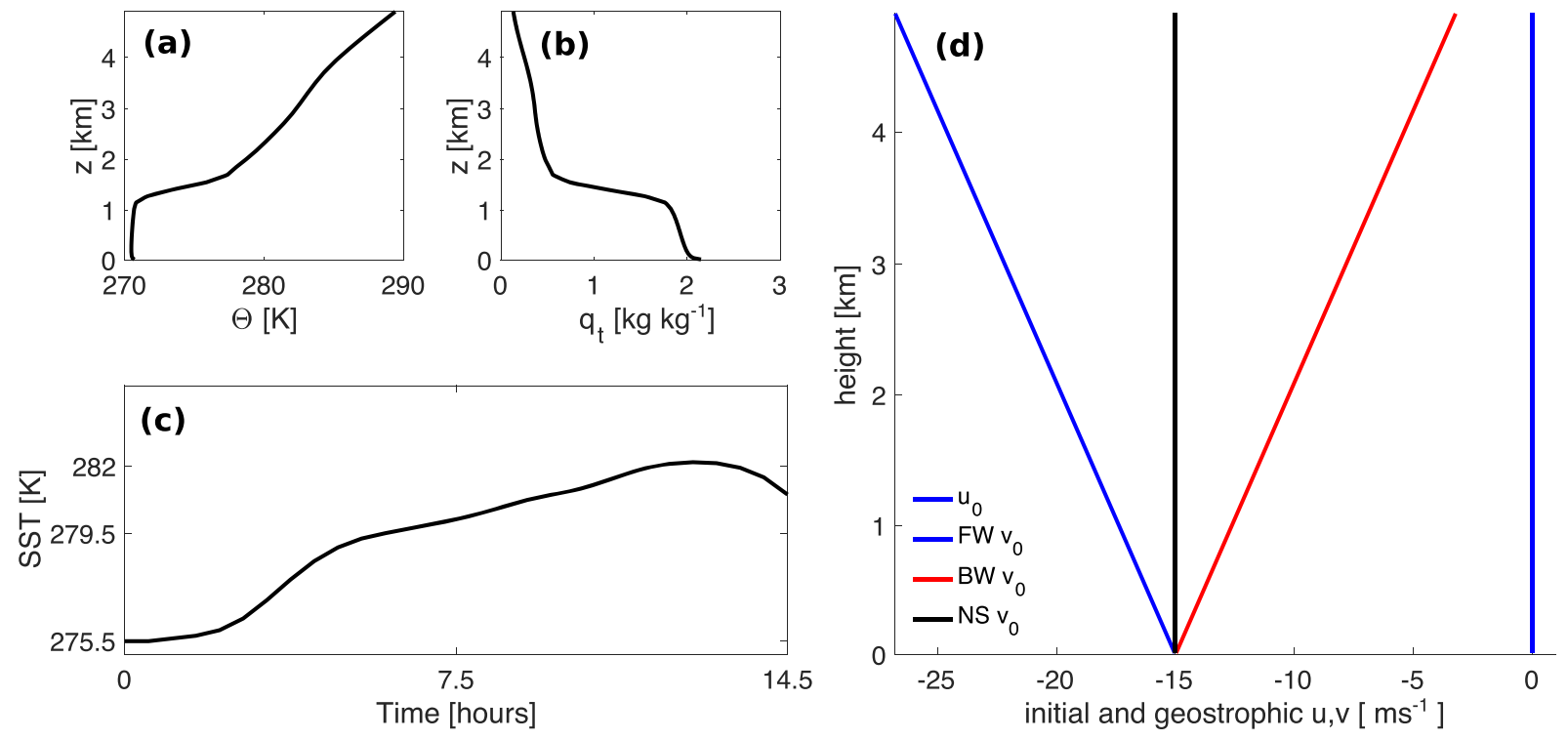

Figure 1. Initial profile of (a) potential temperature and (b) total water specific humidity, and a time varying SST (c). Initial and geostrophic wind profiles of the FW (blue line), the BW (red line), and the NS case (black line) are shown in (d). The simulations only differ in the prescribed meridional wind profiles.

We study this problem by running a Langrangian LES of a well-observed CAO case developed by the WGNE Greyzone project (Field et al., 2014), which we subject to different vertical shear in one of the wind components. By keeping the initial surface wind speed the same, we focus on the effect of momentum transport associated with shear and not on the influence of wind speed itself. In another set of simulations, we completely remove the surface flux response to changes in surface winds that will nevertheless develop, which helps reveal any direct effect of shear on the structure and evolution of turbulence and clouds. In section 2, we describe the setup and methodology of our LESs. In section 3, we discuss some general features of the wind evolution in the $\mathrm{CAO}$ and describe the contribution of momentum transport to the kinetic energy budget. In section 4, we discuss the nature of the momentum transport profiles and the scales at which the transport takes place. Finally, in section 5 we present the evolution of the boundary layer and its clouds under different vertical shear in the geostrophic wind.

\section{Methodology}

\subsection{Case Setup and DALES}

Our analysis is based on turbulence-resolving simulations of the CONSTRAIN case using the Dutch Atmospheric Large Eddy Simulation (DALES) model, which is an open-source LES code developed in the Netherlands (Heus et al., 2010). The CONSTRAIN case setup is based on a CAO trajectory spanning from the Norwegian Sea toward the Atlantic Ocean $\left(66^{\circ} \mathrm{N} 11^{\circ} \mathrm{W}\right.$ to $\left.60^{\circ} \mathrm{N} 8.7^{\circ} \mathrm{W}\right)$ that took place on 31 January 2010. This was a classical CAO, whereby strong meridional flow carried cold air masses southward over increasingly warmer waters, leading to the typical transition from stratocumulus to shallow cumulus clouds. This type of transition is discussed in Brümmer (1997). Using high-resolution limited area model simulations performed with the Met Office Unified Model, an idealized LES case was constructed, which prescribes initial thermodynamic and dynamic profiles and large-scale forcings (Field et al., 2014).

In short, initial profiles of liquid water potential temperature and total water specific humidity are characterized by a well-mixed boundary layer capped by a strong inversion at $\approx 1.5 \mathrm{~km}$ (Figures $1 \mathrm{a}$ and $1 \mathrm{~b}$ ). A time-varying sea surface temperature (SST) is prescribed to mimic a Lagrangian system that moves southward (Figure 1c). This surface forcing and the interactive radiation are the only time-varying forcings we prescribe. Large-scale horizontal advection is ignored and the subsidence is prescribed as a time-varying profile that is only applied to thermodynamics $\left(q_{t}\right.$ and $\left.\theta_{l}\right)$, but not to momentum as to preserve mass continuity, as described in the appendix of De Roode et al. (2019). Hence, in our simulations we decouple the clouds from their large-scale forcing, assuming that the adjustment of the boundary layer to imposed forcing acts on short time scales. This choice is motivated by our desire to gain insight into the role of boundary layer 
processes and somewhat supported by observational and modeling studies, which show that the development of the convective boundary layer embedded in CAOs is largely driven by subsynoptic-scale conditions, such as surface latent and heat fluxes, buoyancy, and cloud top wind shear (Boers \& Melfi, 1987; Boers et al., 1991; Brümmer, 1999; Raasch, 1990; Wayland \& Raman, 1989).

The simulations are run on a domain of $96 \times 96 \mathrm{~km}^{2}$ in the horizontal and $5 \mathrm{~km}$ in the vertical, with a horizontal resolution of $\approx 250 \mathrm{~m}$, and a vertical resolution of $25 \mathrm{~m}$ up to $3 \mathrm{~km}$, which is stretched with a parameter $\alpha=1.02$ in the remaining $2 \mathrm{~km}$. This horizontal resolution is sufficient to capture the closed-and open-cell structure of the cloud deck (Wang \& Feingold, 2009). All simulations are run for $14.5 \mathrm{hr}$, of which the first $2 \mathrm{hr}$ are considered as spin-up time and are not included in the statistics.

A recent intercomparison study discusses the representation of the cloud transition in this CAO as simulated with different LES codes (De Roode et al., 2019). The evolution and timing of the transition from closed to open cells can differ between LES codes. Although all models eventually break up the stratocumulus cloud deck, DALES is particularly slow in performing the transition. Adding ice microphysics and reducing the cloud droplet concentration from 50-100 to $10 \mathrm{~cm}^{-3}$ can speed up the transition (Field et al., 2017). Given the tendency of DALES to postpone the cloud transition, we here set the number of cloud droplets to $10 \mathrm{~cm}^{-3}$ (compared to $50 \mathrm{~cm}^{-3}$ in the original setup). This small number of only $10 \mathrm{~cm}^{-3}$ was observed in the cumulus-dominated phase of the transition (Field et al., 2017). In this study DALES is used with a two-moment bulk microphysics scheme (Seifert \& Beheng, 2001), with an interactive radiation scheme from Fu and Liou (1992), Fu et al. (1997), and Pincus and Stevens (2009), and a hybrid fifth-order advection scheme for momentum and scalars.

\subsection{Wind Shear and Surface Fluxes}

In the original case setup the initial horizontal wind profiles differ from geostrophic balance, which leads to inertial oscillations. As our focus is on the evolution of wind profiles, we wish to avoid these oscillations and therefore use initial wind profiles that are equal to the geostrophic wind profiles (Schlemmer et al., 2016, 2017).

Our prescribed wind profiles are inspired by the climatology of wind shear in marine CAO's derived from ERA-Interim reanalysis data (November to April, from 1979 to 2016, Dee et al., 2011). To compile this climatology, we identified air masses that are substantially colder than the sea surface, that is, $\theta_{\mathrm{SST}}-\theta_{900}>4 \mathrm{~K}$ (Papritz \& Spengler, 2017). Wind shear is considered between the lowest model level ( $\approx 10 \mathrm{mAGL})$ and $800 \mathrm{hPa}$, which is a typical inversion height. Four types of low-level shear are distinguished relative to the wind at $900 \mathrm{hPa}(v)$ : weak shear $\left(\left|\partial_{z} v\right|<1 \mathrm{~ms}^{-1}(100 \mathrm{hPa})^{-1}\right)$, and three cases for $\left|\partial_{z} \nu\right| \geq 1 \mathrm{~ms}^{-1}$ $(100 \mathrm{hPa})^{-1}$, identified by the magnitude of the cosine of the angle between the wind and the wind shear vector $\cos \alpha=\left(\nu \partial_{z} v\right)|\nu|^{-1}\left|\partial_{z} v\right|^{-1}$. Namely, forward shear $\left(\cos \alpha>2^{-1 / 2}\right)$ (see also Figure 1d), backward shear $\left(\cos \alpha<-2^{-1 / 2}\right)$, and lateral shear, where the wind shear vector deviates by more than $45^{\circ}$ from the direction of the background flow $\left(-2^{-1 / 2} \leq \cos \alpha \leq 2^{-1 / 2}\right)$. In the region of the CONSTRAIN case, the wind shear magnitude in the boundary layer ranges from 2 to $4 \mathrm{~ms}^{-1}(100 \mathrm{hPa})^{-1}$. The most common shear is the forward shear (FW) 25-35\%, while the backward shear (BW), weak shear (NS), and lateral shear categories contribute about $10-25 \%$ each (Figure 2). In this study we are predominantly interested in shear in the direction of the background flow and will not consider the lateral shear case.

CAOs are typically embedded in northerly flow to the west of a low-pressure system, with higher pressure to the west (Kolstad et al., 2009; Zolina \& Gulev, 2003). The large-scale shear is to first order determined by thermal wind balance. This is confirmed by composites of $\theta_{900}$ and wind shear for CAO cases in the CONSTRAIN region with predominantly northerly flow at $900 \mathrm{hPa}$. Forward shear cases occur under the influence of a strong low-pressure system in the Norwegian Sea in cyclonically curved flow. Thereby, a tongue of cold air extends to the east of the region such that the thermal wind has a strong southward component and is, thus, aligned with the background flow (Figure 3a). In the backward shear case, in contrast, the CAO is embedded in anticyclonically curved flow with the tongue of cold air extending to the west of the region, resulting in northward directed thermal wind opposing the background flow (Figure 3c). Finally, in the weak shear case the centerline of the CAO air mass is aligned with the CONSTRAIN region (Figure 3b).

We will run the CONSTRAIN case setup with three different profiles of the meridional wind (note that the zonal wind component is 1 order of magnitude smaller than the meridional component for the CONSTRAIN case) that correspond to the FW, NS, and BW shear in the climatology (Figure 1d). The meridional winds 
(a) forward shear

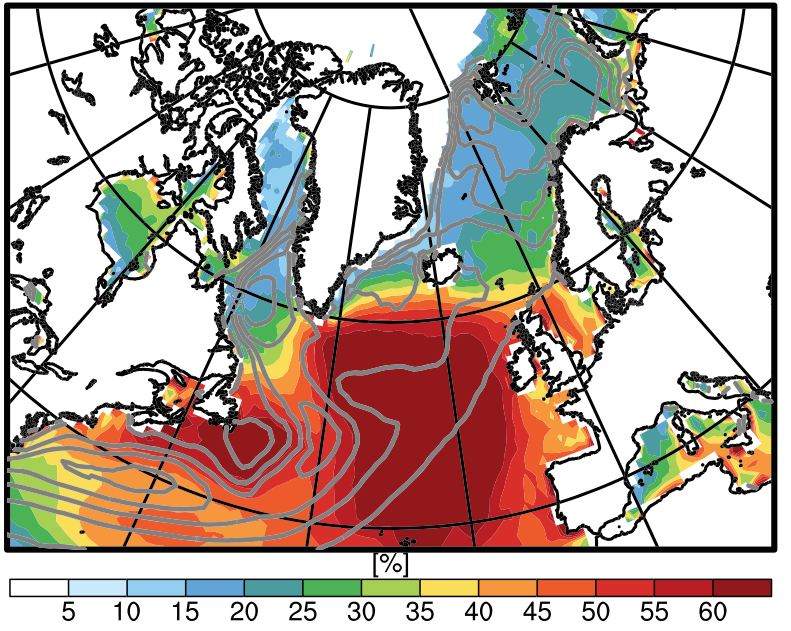

(c) no shear

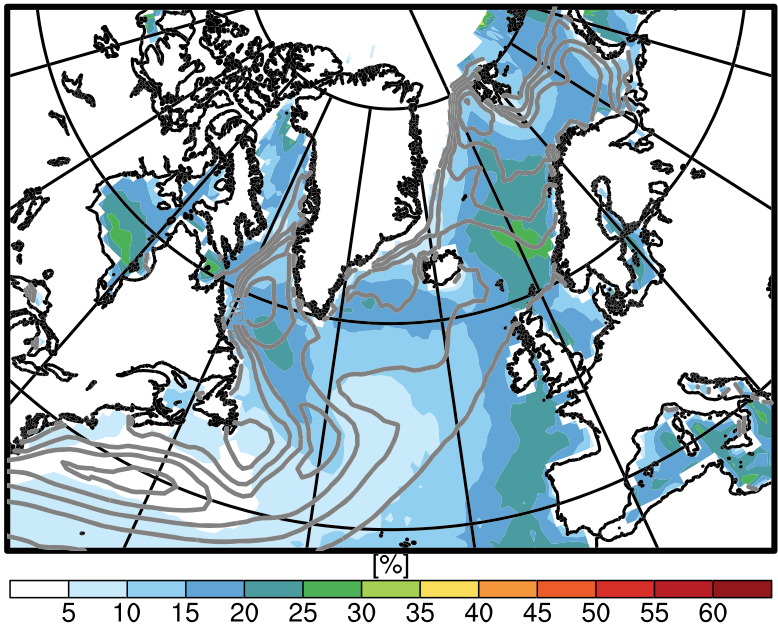

(b) backward shear

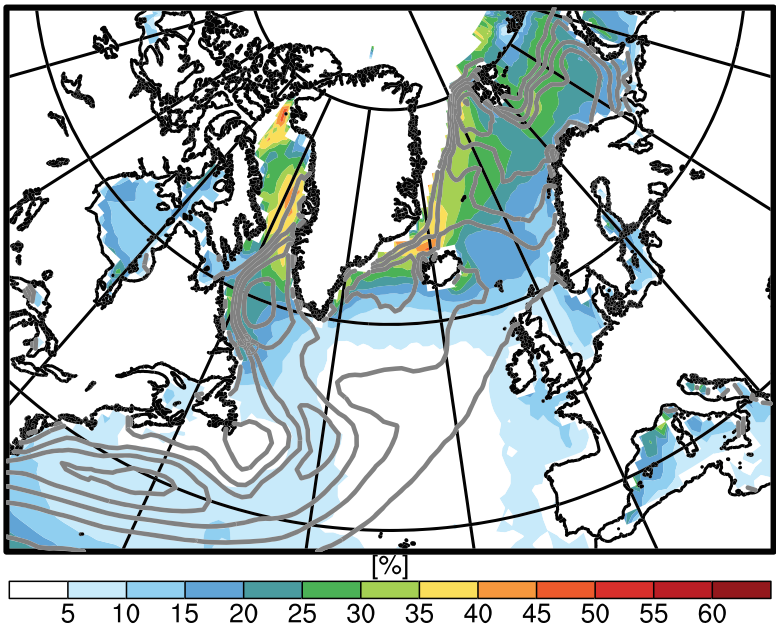

(d)

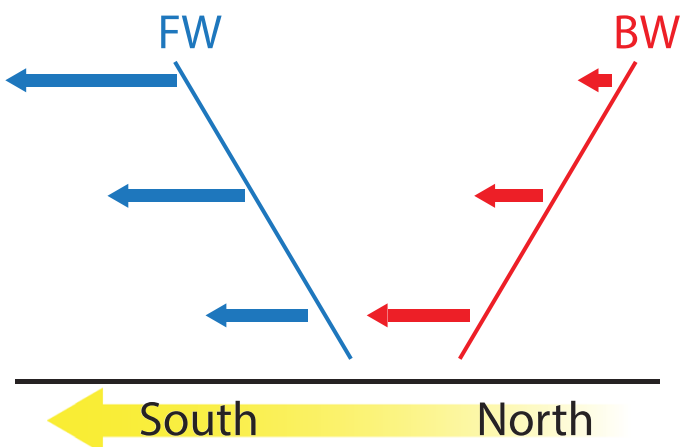

Figure 2. Relative frequency of low-level wind shear type in CAO's. The shear is taken in the layer 800 hPasurf. (a) Forward shear, (b) backward shear, (c) weak shear, (d) schematic of the shear types considered in this study. The frequency of CAO's is indicated by the gray contours in intervals of $10 \%$.

are $-15 \mathrm{~ms}^{-1}$ at the surface and are subjected to a vertical shear of $\pm 2.4 \mathrm{~ms}^{-1} \mathrm{~km}^{-1}$ in the FW and BW shear cases. The zonal wind is initialized to constant zero in all simulations. Galilean transformations are applied to the wind fields to reduce the cross-grid fluxes $\left(-18,-12\right.$, and $-15 \mathrm{~ms}^{-1}$, respectively, in FW, BW, and NS in the meridional direction, and $+2 \mathrm{~ms}^{-1}$ in the zonal direction, because the $u$ wind will quickly evolve from its initial value equal to zero due to Coriolis forces).

The surface fluxes are calculated using standard bulk aerodynamic formulae:

$$
\begin{gathered}
\left.\psi w\right|_{s}=-C_{S}|U|_{s}\left(\psi_{L 1}-\psi_{s}\right), \\
u_{*}=\sqrt{C_{M}}|U|_{S},
\end{gathered}
$$

where $\psi \in\left\{q_{t}, \theta_{l}\right\}$, the subscript $s$ stands for surface, and $L 1$ stands for the first level above the surface, $|U|_{s}$ is the total surface wind speed. The quantities $C_{S}$ and $C_{M}$ depend on the scalar and wind roughness length, respectively. The surface pressure is prescribed at $1,009 \mathrm{hPa}$ and the roughness length for momentum is $z_{0}=6.6 \cdot 10^{-4} \mathrm{~m}$ and for scalars $z_{T}=3.7 \cdot 10^{-6} \mathrm{~m}$.

With this formulation, the surface fluxes are interactive in that they directly depend on the surface wind speed (equations (1), and (2)) and on the near-surface gradient in scalar variable (equation (1)). The larger the surface wind speed, also the larger the momentum flux $u_{*}$ (e.g., the surface stress). We purposely 
(a) forward shear

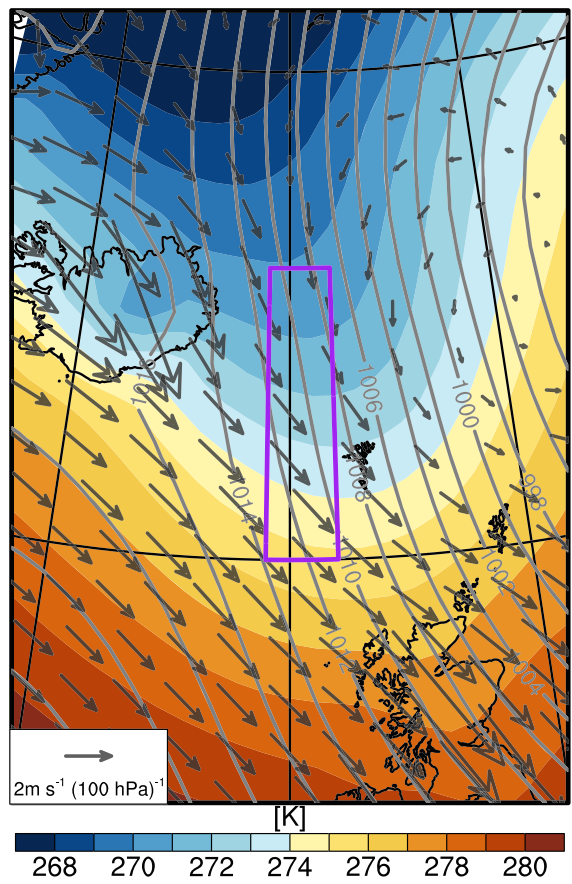

(b) no shear

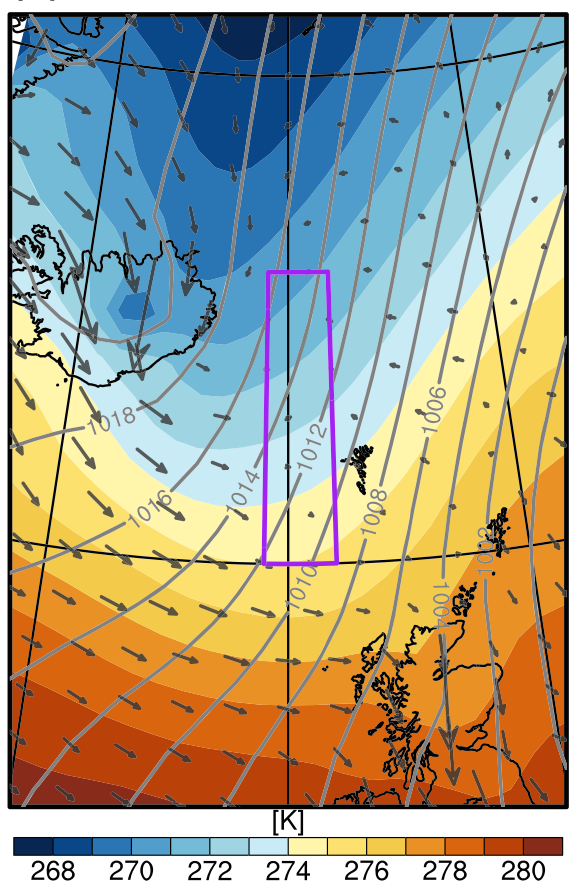

(c) backward shear

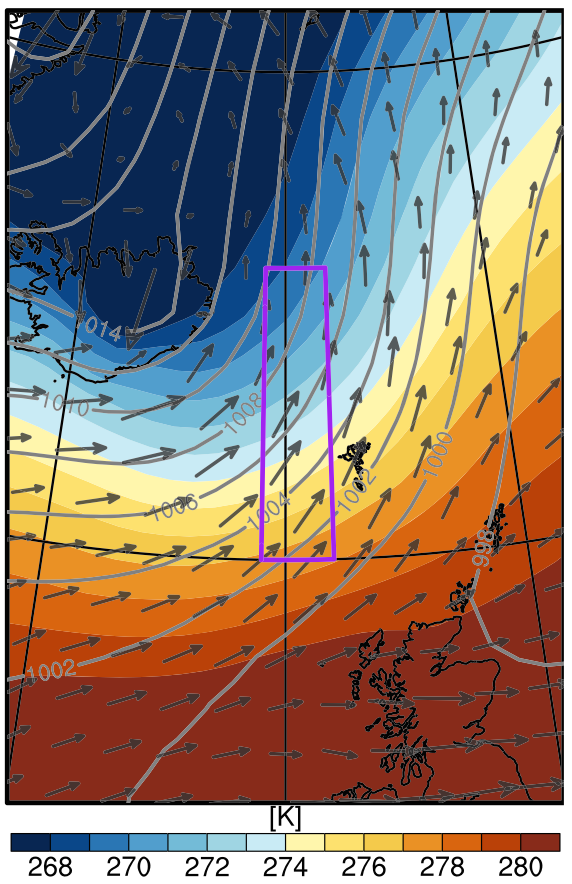

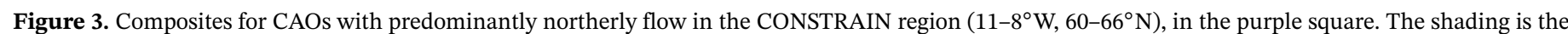
potential temperature at $900 \mathrm{hPa}$, the contours are the sea level pressure, and the arrows are wind shear between $800 \mathrm{hPa}$ and $10 \mathrm{~m}$.

prescribe initial and geostrophic wind profiles that have the same wind speed at the surface, because we are interested in the effect of momentum transport that can be revealed by different shear in the winds, and not the effect of surface wind itself. As our results show, different surface winds and thus different air-sea heat exchanges will develop under different wind shear due to momentum transport, which is an important first-order effect. In section 5 we will explore how the boundary layer evolves differently when we prescribe the surface wind speed in the calculation of the surface heat fluxes (equation (1)).

\section{Wind Turning, Momentum Transport and the Kinetic Energy Budget}

Our first objective is to understand the role of CMT in setting the mean winds in the CAO. Because the clouds and underlying convection undergo a transition during the $14.5 \mathrm{hr}$ of the simulation, during which the airmass is advected over warmer water, we discuss some of the general characteristics of the evolution first and identify different (cloud) phases during the simulation.

\subsection{General CAO Evolution}

In response to increasing SSTs (Figure 1c) the cloud deck transitions from a closed cellular to an open cellular structure, as illustrated in Figure 4, which shows the albedo in the three stages of the transition for the FW shear case. Three phases of the transition can be distinguished: a stratocumulus phase I (Figures $4 \mathrm{a}$ and $4 \mathrm{~d}$ ) from the second to the sixth hour, a transition phase II (Figures $4 \mathrm{~b}$ and $4 \mathrm{e}$ ) from the sixth to the tenth hour, and a cumulus phase III (Figures $4 \mathrm{c}$ and $4 \mathrm{f}$ ) from the tenth hour to the end of the simulation.

All three shear simulations have a similar cloud structure in the first phase, where stratocumuli are present, except that the FW shear case has a slightly higher cloud deck (Figure 4d). In the transition phase, cumulus clouds are forming below the stratocumulus deck, and a higher cloud base and cloud top, and a lower cloud fraction maximum in the FW case are more pronounced. In the last phase, all three cases retain part of the stratocumulus deck, but with the smallest cloud fraction in the FW case.

The mean profiles of horizontal winds, specific humidity, and potential temperature throughout the $14.5 \mathrm{hr}$ simulation are shown in Figure 5 for the three shear cases. Evidently, differences in the mean thermodynamic properties under different wind shear develop, although they remain relatively small. The boundary layer in the FW case is deeper, warmer and more humid. The deeper boundary layer can be explained by the 

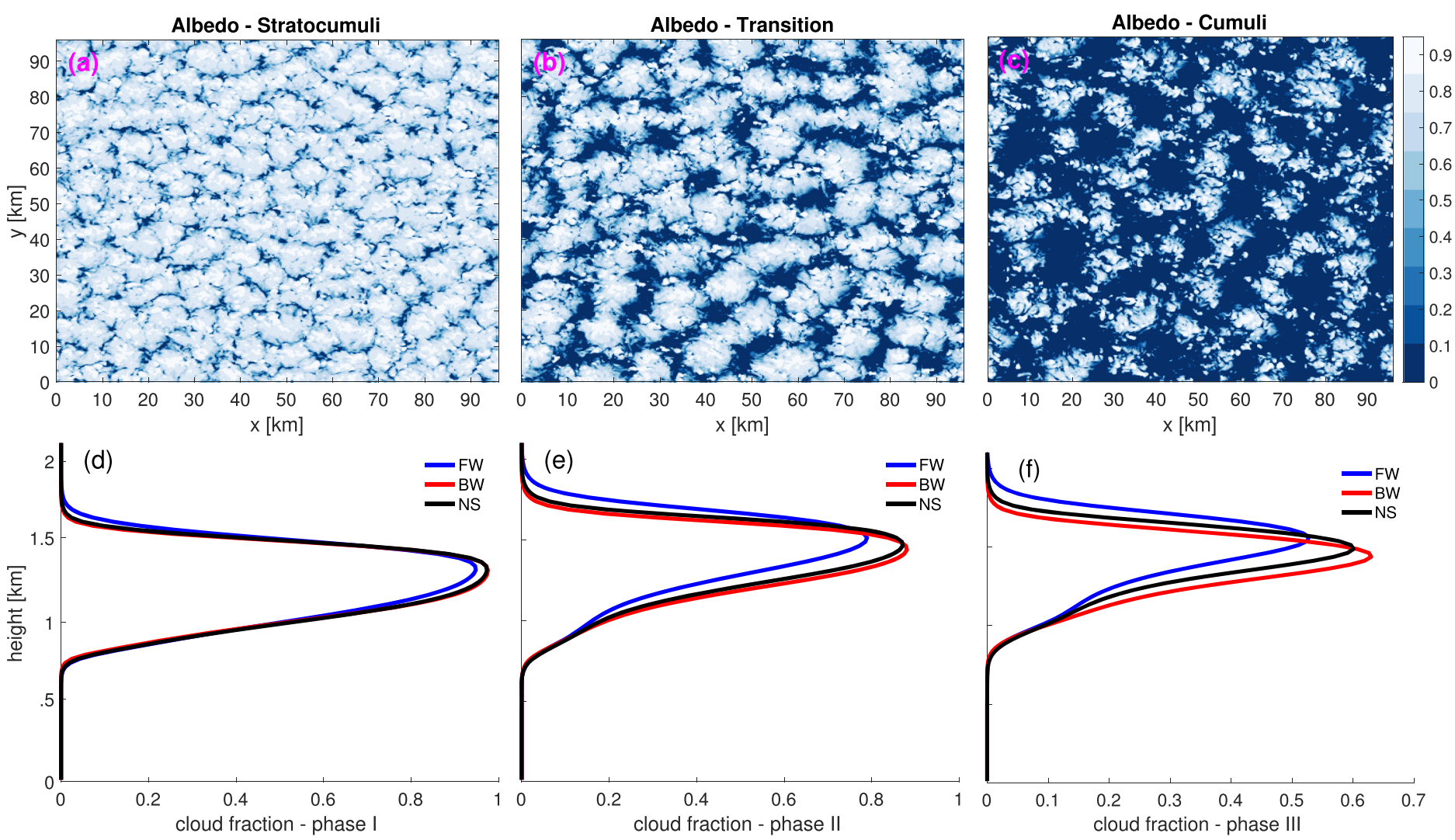

Figure 4. Top row: FW albedo in the stratocumulus phase (a), transition phase (b), and cumulus phase (c). The albedo is defined as $A=\frac{\tau}{6.8+\tau}$, where the optical depth is calculated as $\tau=0.19 L^{5 / 6} N_{\mathrm{c}}^{1 / 3}$, with $N_{\mathrm{c}}$ the cloud-droplet number concentration and $L$ the liquid water path, as in Zhang et al. (2005) and Savic-Jovcic and Stevens (2008). Bottom row (d-f): Cloud fraction for FW (blue line), BW (red line), and NS (black line).

larger surface winds and thus surface fluxes that develop under FW shear. This exemplifies the first-order effect of wind shear that is established through momentum transport. Although the surface winds are the same at the start of the simulation, they evolve differently as momentum transport mixes wind speeds across the boundary layer. This mixing is very efficient, as revealed by the constancy of the meridional wind with height throughout the boundary layer in the three different shear cases.

In section 5 we return to the small but notable differences in the thermodynamic evolution of the CAO. First, we will focus on the evolution of the wind profiles and the resulting wind turning, and ask which processes influence this evolution.

\subsection{Sensitivity of Wind Turning to Shear}

The meridional winds near the surface are about $1,2.5$, and $3.5 \mathrm{~ms}^{-1}$ slower than their geostrophic value of $15 \mathrm{~ms}^{-1}$ in the FW, NS, and BW case, respectively. The zonal winds are 1,2 , and $1.5 \mathrm{~ms}^{-1}$ faster than their initially zero values. The interplay between frictional forces and the Coriolis force helps explain why the zonal wind component develops (equation (5)). Initially, the mean flow is purely southward, but frictional forces, including surface drag, will immediately establish a wind component perpendicular to the mean flow and across isobars, toward the region with lower pressure. In this case, a positive zonal wind develops (see also Figure 6). This effect is stronger in the subcloud layer, as can be seen in Figure 6a. In the FW shear case, vertical mixing will bring stronger meridional winds toward the surface. This leads to less wind turning and thus weaker zonal winds at the surface (Figure 5a). Near the top of the boundary layer, the opposite effect is seen: the upward mixing of relatively slow meridional winds in the FW case, leads to greater wind turning than in the NS and BW cases. In real atmospheres, the CAO system as a whole may not be turning in such a way, because our simulations ignore one important forcing: the large-scale horizontal advection of momentum. We presume that this horizontal momentum advection could largely counteract the strong 

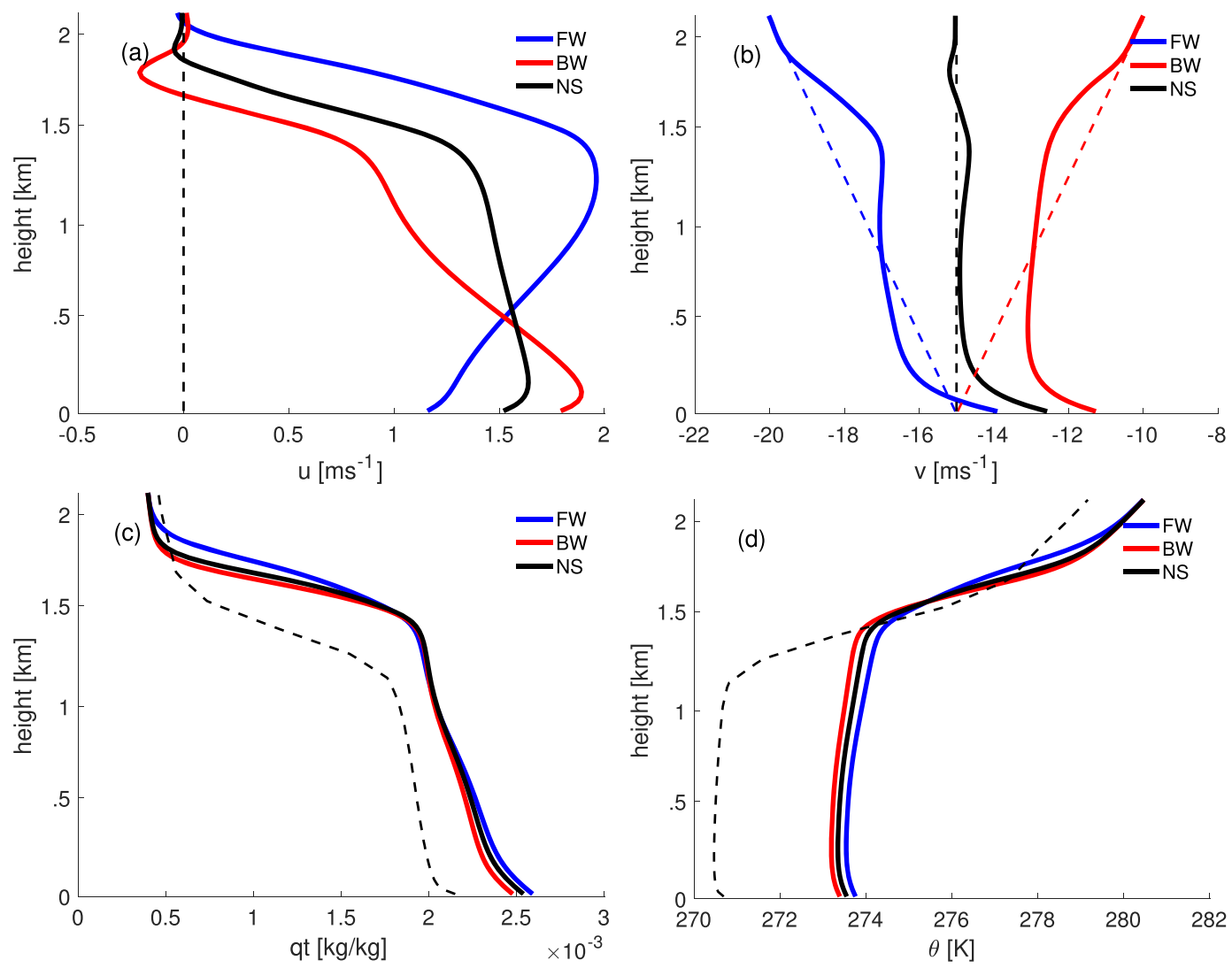

Figure 5. Mean profiles of (a) zonal and (b) meridional wind, (c) total water specific humidity $q_{t}$ and (d) liquid potential temperature $\theta_{l}$ averaged over the whole simulations. The dashed lines represent the initial profiles. The cloud layer extends from 1 to $2 \mathrm{~km}$.

turning at this latitude. Hence, our simulations serve to exemplify the effects introduced by momentum mixing in the presence of a strong Coriolis effect.

This analysis is also important for understanding the cause of the stronger than geostrophic winds that develop. For instance, in the lower part of the boundary layer under FW shear, the meridional wind component is larger than geostrophic, which also leads to stronger total wind speeds. Is this the result of the
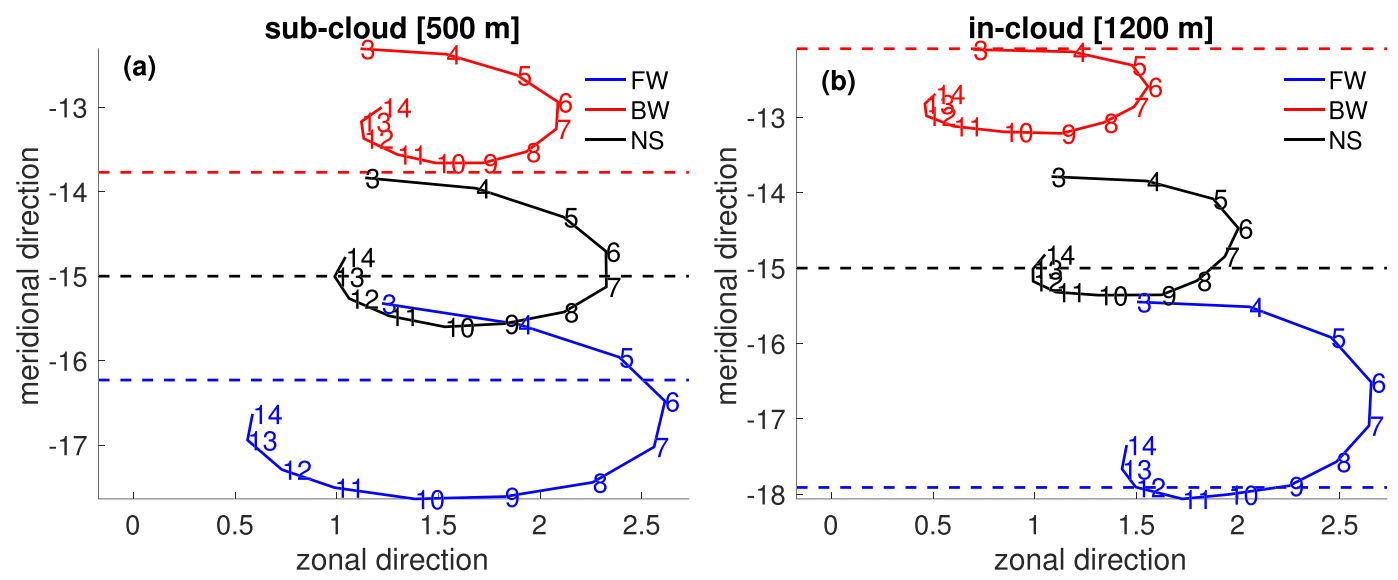

Figure 6. Wind turning in (a) the subcloud layer and in (b) the cloud layer. The numbers are the hours in the simulations. The dotted lines indicate the geostrophic wind at the given height. Inertial oscillations are established due to the Coriolis force. 

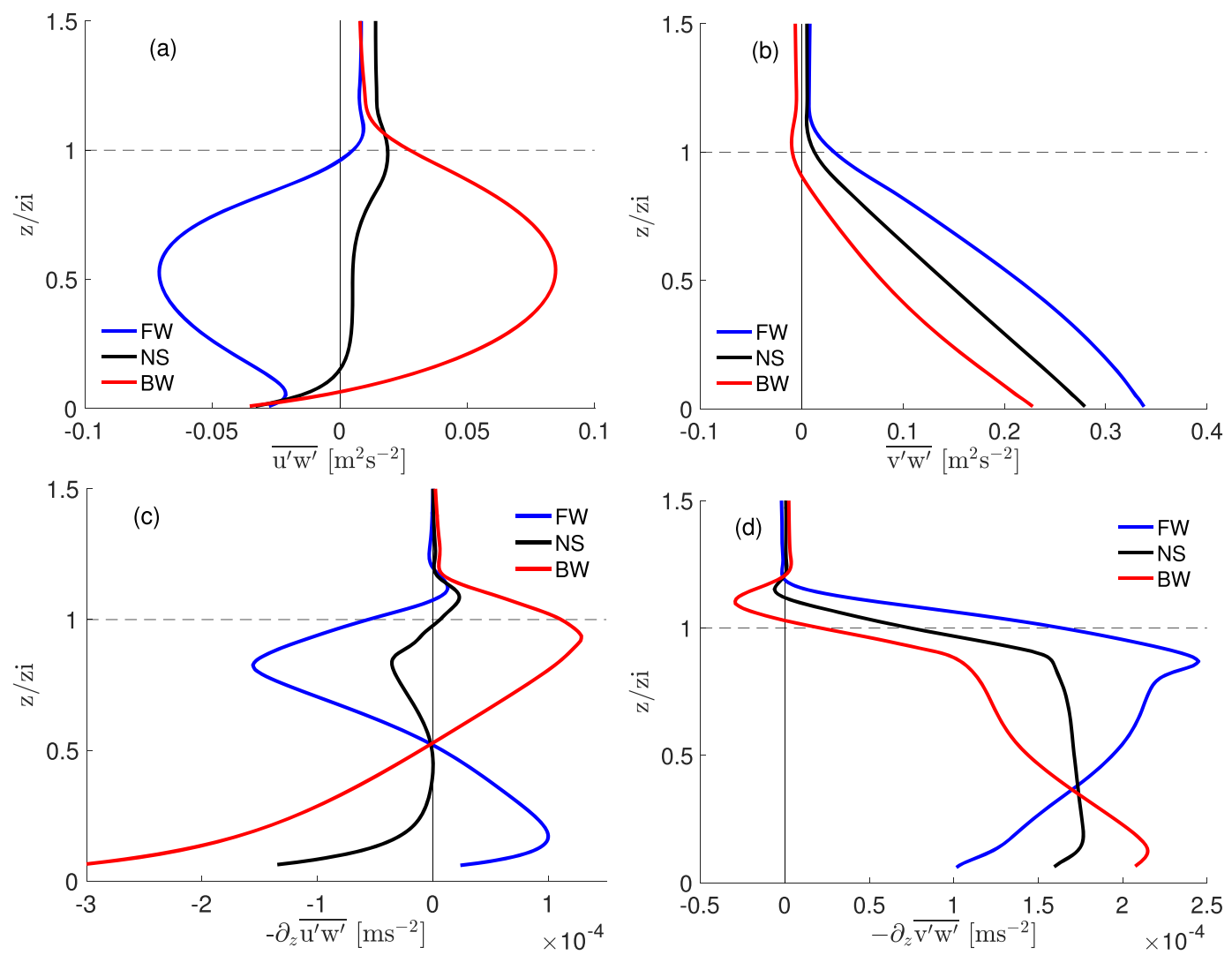

Figure 7. Zonal (a) and meridional (b) momentum fluxes (resolved + subgrid) and their divergence (c, d) averaged over the whole simulation. The $y$ !axis is normalized by the boundary layer height.

downward transport of stronger meridional winds that exist in the upper boundary layer? To answer this question, it is useful to explore the kinetic energy budget and the role of momentum transport therein.

\subsection{The Role of Momentum Transport in the Kinetic Energy Budget}

The tendencies introduced by momentum transport-accelerations or decelerations-can be derived from the profile of zonal and meridional momentum flux (Figures 7a and 7b). In the NS case, the meridional momentum transport profile $\overline{v^{\prime} w^{\prime}}$, which includes both resolved motions and the parameterized subgrid motions in LES, linearly decreases from the surface to zero at the top of the boundary layer. Such a linearly decreasing profile is well-known for clear convective boundary layers (Conzemius \& Fedorovich, 2006; Fedorovich \& Conzemius, 2008) and translates to a constant deceleration of the meridional flow throughout the entire boundary layer (Figure 7d). Under FW and BW shear the meridional momentum flux $\overline{v^{\prime} w^{\prime}}$ also decreases with height, albeit with some more concavity in the profiles. This nonlinear feature modifies the impact of momentum transport in the momentum budget, depending on the height (Figure 7d), which we explore further below by studying the kinetic energy budget. The profiles of the zonal momentum flux $\overline{u^{\prime} w^{\prime}}$ show even larger dependence on the background wind (Figure 7a). These concave momentum fluxes profiles are a result of momentum transport that acts to remove the shear in the zonal wind profiles (Figure 7c), which are never well-mixed due to the different wind turning present at different heights.

The horizontal momentum budget may be written as follows:

$$
\begin{gathered}
\partial_{t} \bar{u}+\bar{u} \partial_{x} \bar{u}+\bar{v} \partial_{y} \bar{u}+\bar{w} \partial_{z} \bar{u}=-\partial_{x} \overline{u^{\prime} u^{\prime}}-\partial_{y} \overline{u^{\prime} v^{\prime}}-\partial_{z} \overline{u^{\prime} w^{\prime}}+f\left(\bar{v}-v_{g}\right), \\
\partial_{t} \bar{v}+\bar{u} \partial_{x} \bar{v}+\bar{v} \partial_{y} \bar{v}+\bar{w} \partial_{z} \bar{v}=-\partial_{x} \overline{v^{\prime} u^{\prime}}-\partial_{y} \overline{v^{\prime} v^{\prime}}-\partial_{z} \overline{v^{\prime} w^{\prime}}-f\left(\bar{u}-u_{g}\right),
\end{gathered}
$$

where the overbars represent mean states, primes are deviations from the mean states, $u_{g}$ and $v_{g}$ are the geostrophic wind components and $f$ is the Coriolis parameter. Ignoring horizontal and vertical advection 

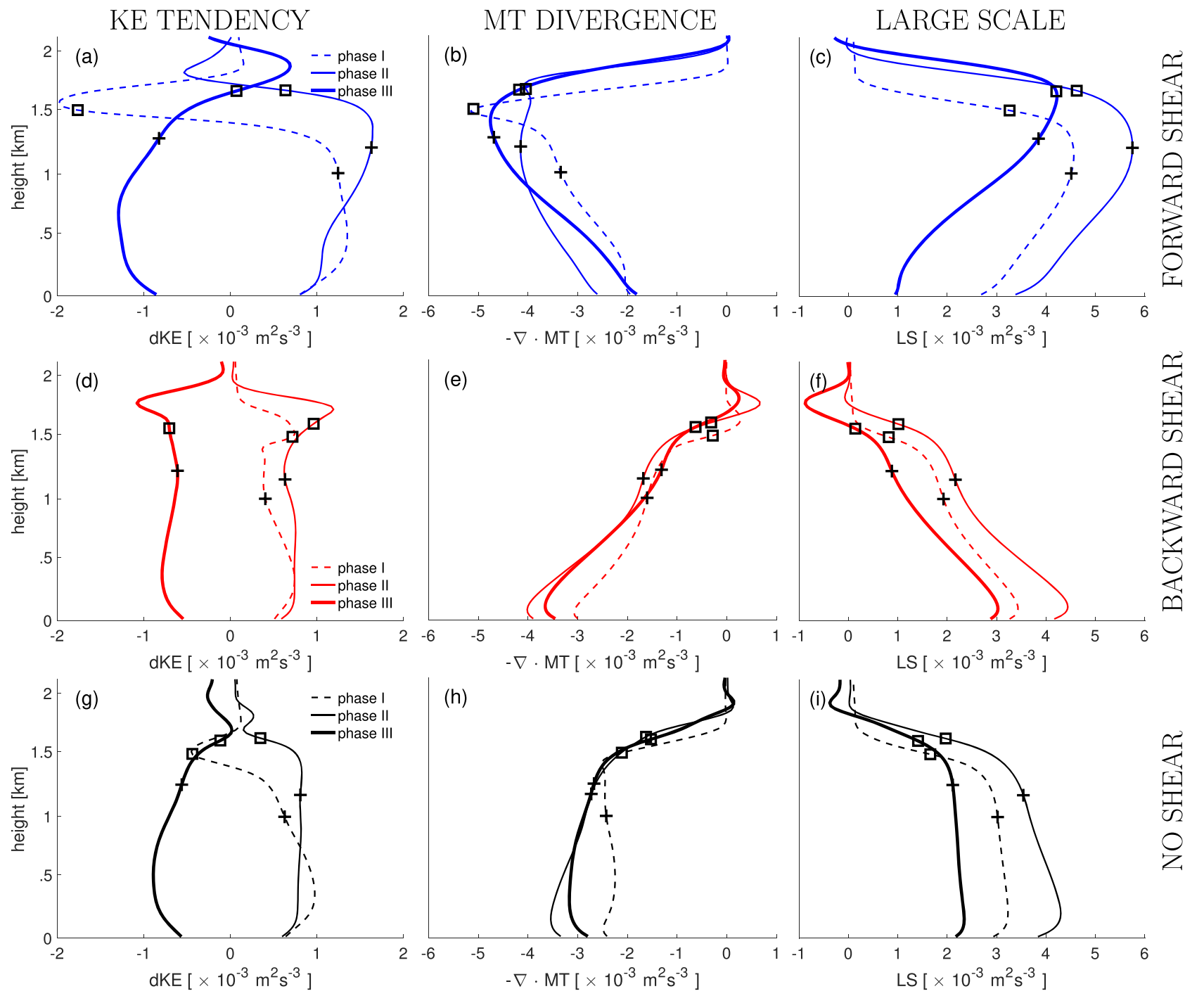

Figure 8. Kinetic energy (KE) budget. (a, d, g) KE tendency, (b, e, h) momentum transport term, (c, f, i) large-scale term. Dashed lines represent the stratocumulus phase, thin lines the transition phase, and thick lines the cumulus phase. The crosses indicate cloud base, and the squares represent cloud top. Blue lines are the FW, red lines are the BW, and the black lines are the NS case.

of momentum (see also section 2.1) and assuming horizontal homogeneity so that the first terms on the right-hand side are approximately zero, the horizontal momentum budget becomes

$$
\begin{aligned}
& \partial_{t} \bar{u}=-\partial_{z} \overline{u^{\prime} w^{\prime}}+f\left(\bar{v}-v_{g}\right), \\
& \partial_{t} \bar{v}=-\partial_{z} \overline{v^{\prime} w^{\prime}}-f\left(\bar{u}-u_{g}\right),
\end{aligned}
$$

The first term on the right-hand side is the momentum transport divergence. The terms $f\left(\bar{v}-v_{g}\right)$ and $-f\left(\bar{u}-u_{g}\right)$ are the combination of the Coriolis force and the large-scale pressure gradient, also called the "ageostrophic component." The kinetic energy is defined as $\mathrm{KE}=\frac{1}{2}\left(\bar{u}^{2}+\bar{v}^{2}\right)$, whose tendency is $\partial_{t}(\mathrm{KE})=\bar{u} \partial_{t} \bar{u}+\bar{v} \partial_{t} \bar{v}$. The KE budget equation is derived by multiplying the first equation by $\bar{u}$ and the second by $\bar{v}$ and then summing the two equations. When doing so, the Coriolis forcing terms cancel out, leaving the momentum transport and the pressure gradient terms as follows:

$$
\partial_{t}(\mathrm{KE})=-\left(\bar{u} \partial_{z} \overline{u^{\prime} w^{\prime}}+\bar{v} \partial_{z} \overline{v^{\prime} w^{\prime}}\right)+\left(-f \bar{u} v_{g}+f \bar{v} u_{g}\right)
$$

The first term in parentheses on the right-hand side is the momentum transport contribution to the kinetic energy, and the second term in parentheses is the large-scale pressure gradient contribution. In these simulations $u_{g} \equiv 0$, and $\bar{u} \partial_{z} \overline{u^{\prime} w^{\prime}}$ is one order of magnitude smaller than $\bar{v} \partial_{z} \overline{v^{\prime} w^{\prime}}$. Therefore, the KE budget may 

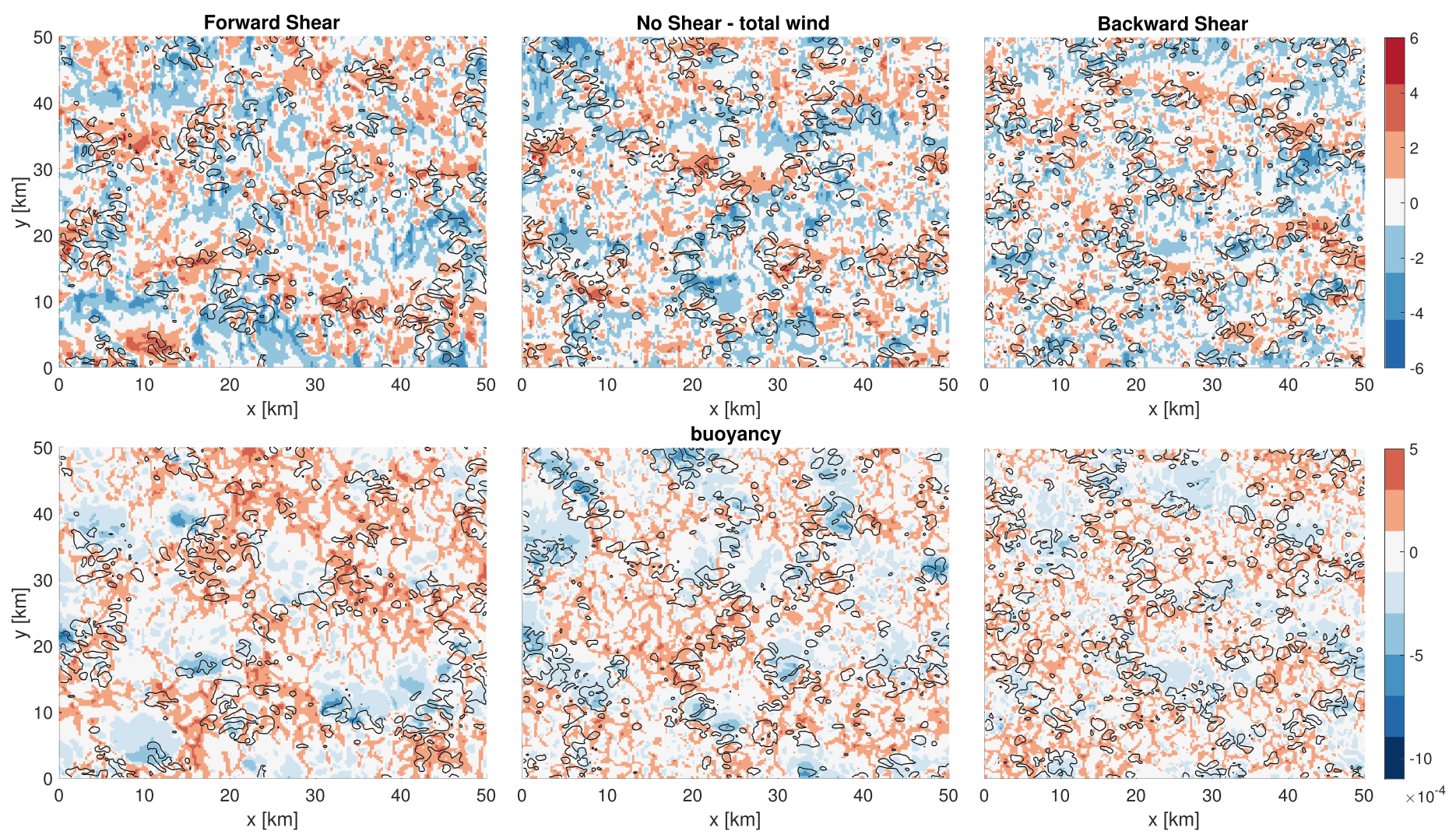

Figure 9. Total wind field perturbation $\left[\mathrm{ms}^{-1}\right]$ (first row) and buoyancy $[\mathrm{K}]$ (second row) at $50 \mathrm{~m}$ for FW, NS, and BW, respectively at the end of the simulation. The black lines are liquid water contours at $2 \cdot 10^{-4} \mathrm{~kg} \cdot \mathrm{kg}^{-1}$ at $1.5 \mathrm{~km}$.

be approximately written as

$$
\partial_{t}(\mathrm{KE}) \approx-\bar{v} \partial_{z} \overline{\nu^{\prime} w^{\prime}}-f \bar{u} v_{g}
$$

Figure 8 shows the kinetic energy budget profiles (equation (8)) for each simulation, with from left to right, the KE tendency, the momentum transport (MT) term and the large-scale pressure gradient term. The terms are shown for three phases of the transition, which reflects the oscillation of winds, whereby kinetic energy production first increases and then decreases.

The momentum transport divergence $-\nabla \cdot$ MT acts to oppose the large-scale increase in kinetic energy (Figures 8b, 8e, and 8h). The generation of kinetic energy takes place as the winds turn across isobars away from the imposed geostrophic wind direction, in other words, through the interaction of zonal wind $u$ with the geostrophic wind $\mathrm{v}_{\mathrm{g}}$ (the second "large-scale" term on the right-hand side of equation (8), in the rightmost panels in Figure 8). The presence of the zonal wind can be thought of as a reservoir of momentum for the meridional flow. The wind speeds that become stronger than geostrophic near the surface in the FW case or near cloud tops in the BW case (Figure 5b) are thus a result from the energy created as cross isobaric flow or ageostrophic winds arise. In fact, since $u_{g} \approx-\partial_{y} p$ and $v_{g} \approx \partial_{x} p$, the term $-f \bar{u} v_{g}+f \bar{v} u_{g}$ is the work done by the pressure gradient force,

$$
-f \bar{u} v_{g}+f \bar{v} u_{g} \approx-f \bar{u} \partial_{x} p-f \bar{v} \partial_{y} p
$$

The effect of the Coriolis force is then to induce the turning of the zonal wind component in the meridional direction.

Momentum transport introduces a deceleration in all cases (with the exception of a few hundred meters near cloud tops in the BW shear case). In the NS case, the contribution by momentum transport is mostly constant throughout the layer during each phase. For the FW shear case, the friction introduced by MT is much larger in the cloud layer $\left(-5\right.$ to $\left.-4 \mathrm{~ms}^{-3}\right)$, whereas in the BW shear case the sub-cloud layer experiences more friction $\left(-4\right.$ to $\left.-3 \mathrm{~ms}^{-3}\right)$. 

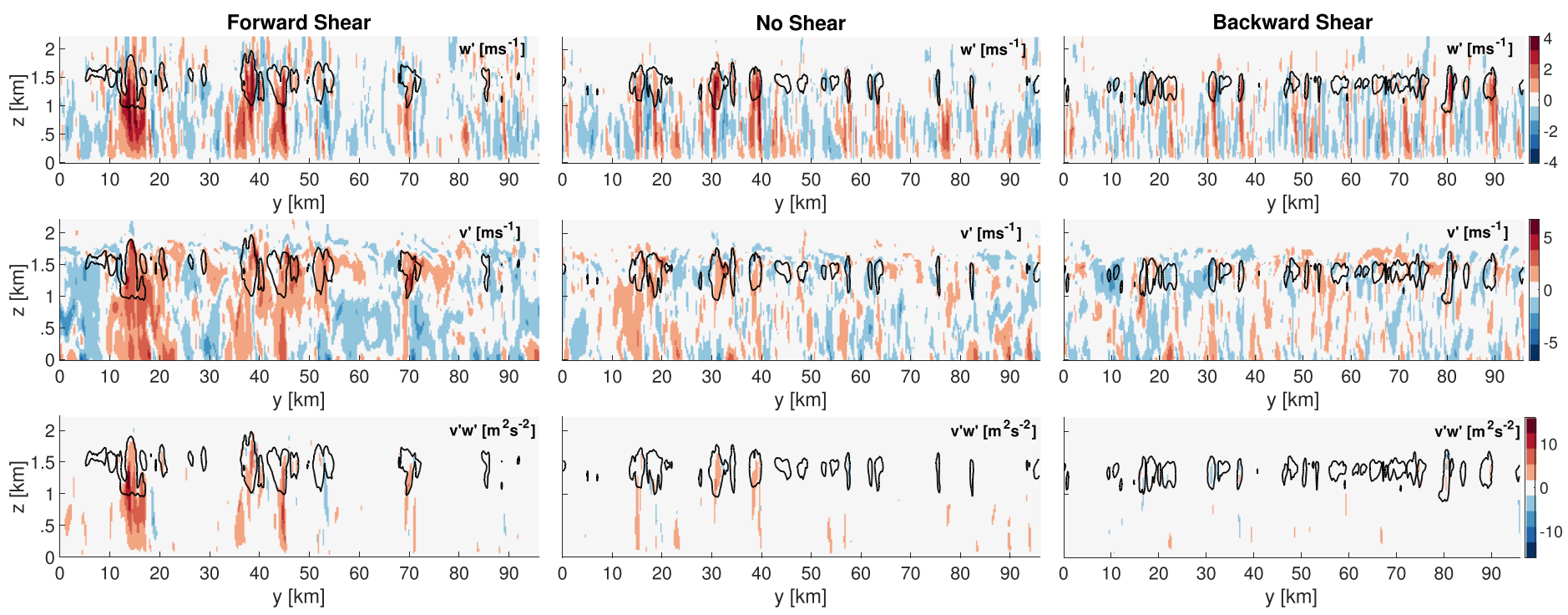

Figure 10. The $x-z$ snapshots of vertical velocity anomalies $w^{\prime}$ (top row), meridional wind anomalies $v^{\prime}$ (middle row), and vertical flux of meridional momentum $v^{\prime} w^{\prime}$ (bottom row) at hour 14.5. The black lines are the clouds contours.

In all cases, as the inertial oscillations cause the flow to approach geostrophic balance, the production of kinetic energy through the cross-isobaric flow will be reduced (such as in the third phase). The friction induced by MT then dominates, slowing down the CAO airmass (a negative KE tendency, Figures 8a, 8d, and $8 \mathrm{~g})$.

\section{Role of Shallow Convection in Momentum Transport}

In this section, we address how turbulence, respectively, convective motions help shape the MT profile and how these contributions change with shear. The momentum transport profile depends on the distribution of the vertical velocity, updrafts, and downdrafts and their intensity and location, and on the distribution of the horizontal winds with respect to this structure. The scales of variability involved in setting the momentum flux is rich, as illustrated in Figure 9, which displays the total wind field perturbation $\mathrm{U}^{\prime}$, where $\mathrm{U}=\sqrt{u^{2}+v^{2}}$ is the total wind and $\mathrm{U}^{\prime}=\mathrm{U}-\overline{\mathrm{U}}$, as well as the buoyancy field at $z=50 \mathrm{~m}$ at the end of the simulation for the FW, NS, and BW cases. The black lines are cloud contours at $q_{l}=2 \cdot 10^{-4} \mathrm{~kg} \cdot \mathrm{kg}^{-1}$ at $1.5 \mathrm{~km}$. For the sake of clarity, we zoomed in on a $50 \times 50 \mathrm{~km}^{2}$ subdomain.

The snapshots reveal the pronounced mesoscale circulations between the closed and open cells present in the cloud field. These circulations develop due to horizontal heating gradients, and are generally thought to be caused by evaporation of precipitation (cold pools), radiative and surface flux feedbacks (Muller \& Bony, 2015; Seifert \& Heus, 2013; Wang \& Feingold, 2009), or water vapor-convection feedbacks (Bretherton \& Blossey, 2017). The near-surface horizontal wind fields are characterized by divergence and convergence patterns of the cold pools. Because the FW shear case develops a deeper boundary layer and more precipitation (see section 5), the cold pools here are stronger and larger than in the NS and BW shear cases (areas of negative buoyancy in Figure 9, second row). For example, one may consider the cold pool at $x=10 \mathrm{~km}$, $y=10 \mathrm{~km}$ in the FW shear case. The horizontal wind in the corresponding region shows two diverging patches of faster (red) and slower (blue) winds, where faster here means a stronger southward moving flow. Within these larger wind structures, also many smaller wind variations can be seen, where wind gradients can be up to $6 \mathrm{~ms}^{-1}$ within few kilometers.

The momentum flux profiles that correspond to such variability are illustrated using $x-z$ snapshots during the cumulus cloud regime (phase III) taken at $y \approx 48 \mathrm{~km}$, see Figure 10. The snapshots show, from top to bottom: the vertical velocity anomalies (from the domain mean), the meridional wind anomalies, and the meridional momentum flux. The updrafts in the subcloud layer are linked to cumulus clouds overhead and can have peaks up to $4 \mathrm{~ms}^{-1}$, while downdrafts are weaker, -1 to $-2 \mathrm{~ms}^{-1}$, and localized in the clear sky regions and at the clouds' edges. Stronger and wider updrafts are visible in the FW case, which develop larger cold pools and stronger convergence (Figure 9) and lead to deeper and fatter clouds compared to the NS and 

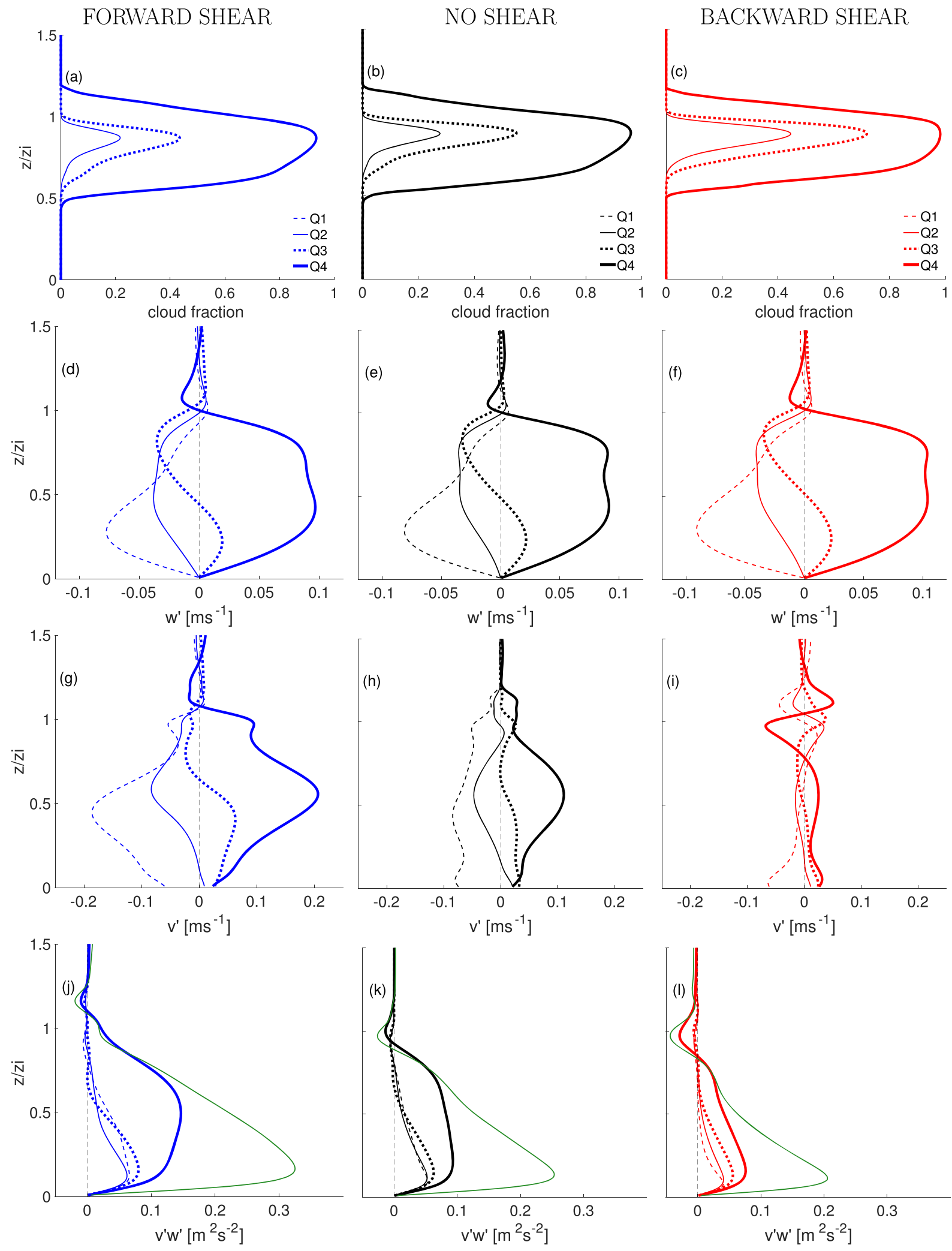

Figure 11. Decomposition in quartiles of CWV of the (a-c) cloud fraction, (d-f) $w^{\prime},(\mathrm{g}-\mathrm{i}) v^{\prime}$, and (j-1) the resolved $v^{\prime} w^{\prime}$ over the last two hours of the simulation. The green lines are the resolved momentum fluxes (which also is the sum of the quartiles). The $y$ axis is normalized by the boundary layer height. 


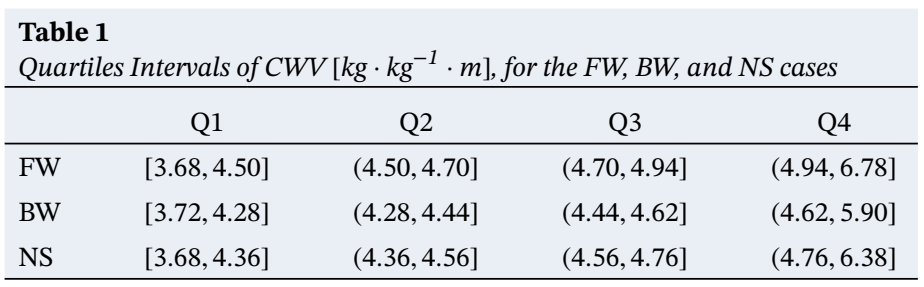

BW shear cases. Evidently, the regions with strong updrafts have a meridional wind that is slower than the mean flow (red), as air from near the surface is transported upward (middle row of Figure 10). Therefore, winds within clouds and within the cloud layer generally tend to be moving slower than the mean airmass. This effect is less clear for the NS and BW shear cases, in which wind speeds in the cloud layer are much closer to wind speed near the surface. Strong cancelations in the product of $w^{\prime}$ and $v^{\prime}$ occur, so that the momentum flux itself (bottom row) is only strongly pronounced in the updraft areas underneath and within clouds. In other words, the bulk of the positive vertical flux of meridional momentum seems carried by the areas with strong moist convection, but they also have a much smaller statistical weight, as they occupy a small portion of the domain (see the $v^{\prime} w^{\prime}$ snapshots in Figure 10). These cross sections represent only a small part of the domain. In the following we attempt to quantify the overall contributions of convective motions by sampling on column water vapor. Additionally, we perform a spectral analysis on the 3-D turbulence fields to quantify the contribution of different scales to the momentum flux.

\subsection{Wind and Momentum Flux Sampled on Column Water Vapor}

Here we average the momentum fluxes over different parts of the domain ordered by their column water vapor (CWV, defined as the integral of $q_{t}$ in the vertical direction), where we assume convective and cloudy regions tend to be the moistest regions within the domain. This method also gives statistical weight to these regions, an aspect that cannot be truly appreciated by looking at the snapshots. The distributions in CWV range from 3 to $7 \mathrm{~kg} \cdot \mathrm{kg}^{-1} \mathrm{~m}$ with intervals $\Delta \mathrm{CWV}=0.02 \mathrm{~kg} \cdot \mathrm{kg}^{-1} \mathrm{~m}$ (the density is not included).

Figure 11 shows slab averages of quartiles of momentum fluxes $v^{\prime} w^{\prime}$ (only the resolved fluxes) and of wind profiles $v^{\prime}$ ordered in such manner, in the last two hours of the simulation (during the cumulus phase III). We identify four quartiles of CWV (based on the frequency of occurrence), as in Table 1.

The first quartile (Q1) contains what we assume are mostly clear sky regions, as revealed from the cloud fraction profiles over just these columns (Figures 11a-11c). The remaining quartiles contain regions with clouds, and the forth quartile (Q4) represents mostly columns with clouds, as the cloud fraction approaches $100 \%$ (Figures 11a-11c). We shall refer to Q2 and Q3 as the environment regions of convective updrafts and to $\mathrm{Q} 4$ as the cloudy region.

The quartiles of vertical velocity show that CWV nicely separates the areas of strong versus weak convection. They also show that vertical velocity distributions are not strongly affected by the wind shear (Figures 11d-11f), different from what might be expected for more vigorous convection, where wind shear itself has long been shown to help organize deep convective cells into storms and squall lines (Weisman \& Klemp, 1984).

The first (mostly clear sky regions), second, and third (partially cloudy regions) quartiles have positive momentum fluxes throughout the layer, which are generally linearly decreasing, and do not extend much in the cloud layer. In these regions the meridional flow experiences a deceleration due to momentum transport. They account for $\approx 60 \%$ of the flux in the subcloud layer ( $\approx 20 \%$ each). The fluxes of Q1 and Q2 are established by strong and weak downdrafts, respectively (Figures 11d-11f), which carry large and small negative meridional wind anomalies (which here means stronger than mean meridional flow; Figures 11g-11i). The downward transport in Q2 is larger in the cloud layer and it is located at the edges of the clouds (Figures 11a-11c), in accordance with the study in Heus and Jonker (2008), but it does not contribute much to the total flux.

The strongly convecting moist areas (Q4) dominate the meridional momentum flux (Figures 11j-111) compared to the drier areas with weakly or strongly subsiding motions (Figures 11d-11f). In fact, Q4 contributes more than $30 \%$ of the flux in the subcloud layer, and for almost all of it in the cloud layer. In Q4 the momentum flux profile no longer decreases with height under FW shear, or much less so under NS or BW shear. 

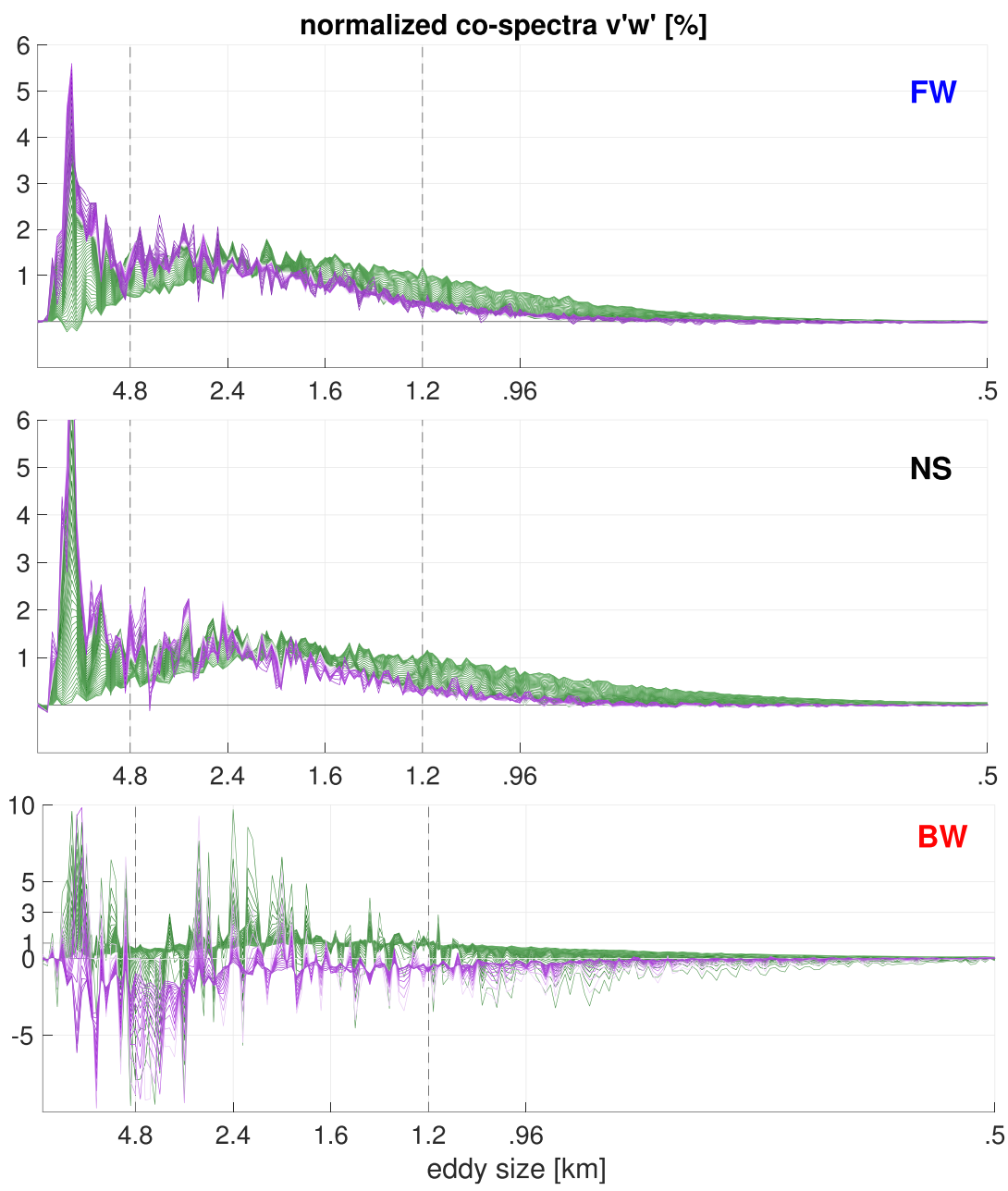

Figure 12. Normalized cospectra representing the resolved fluxes $v^{\prime} w^{\prime}$ in the last two hours of the simulations (cumulus phase); (top) FW, (middle) NS, (bottom) BW. Green lines: from surface to $800 \mathrm{~m}$. Purple lines: from 800 to $1,600 \mathrm{~m}$. The smallest eddy size, $0.5 \mathrm{~km}$, corresponds to twice the grid spacing. The largest eddy size, $48 \mathrm{~km}$, corresponds to half the domain size.

Under FW shear large positive anomalies (slower wind) found in the cloud layer (or near $1 \mathrm{~km}$ ) lead to a weaker decrease in momentum flux at those levels (Figures 11j-111). In other words, the momentum transport carried by convective updrafts tend to accelerate winds in the subcloud layer. This is less true, but still evident for the NS case. It exemplifies how important the shear profile is for understanding the tendency introduced by momentum transport.

\subsection{Momentum Flux Contribution by Scale}

The variety of scales involved in the structure of the horizontal winds (Figure 9) raises the question, which scales contribute the most to the momentum fluxes, and in which way. This issue has also been investigated by Zhu (2015), with the purpose of assessing the validity of the mass flux approach for momentum transport. A powerful technique to investigate this is the 2-D Fourier transform of the horizontal and vertical wind fields. Following Parseval's theorem, as explained by Zhu (2015), the momentum fluxes $\overline{u^{\prime} w^{\prime}}\left(\overline{v^{\prime} w^{\prime}}\right)$ are integrated over all wave numbers of the cospectra of the Fourier transform of $u^{\prime}\left(v^{\prime}\right)$ and $w^{\prime}$. The Fourier analysis is performed on the 3-D fields (2-D horizontal fields at each height level), which are collected every $30 \mathrm{~min}$ for a period of $2 \mathrm{hr}$ during the cumulus phase (III).

Figure 12 shows the normalized cospectra (hence the probability density function) of $v^{\prime}$ and $w^{\prime}$ as a function of decreasing eddy size for FW, NS, and BW. The green lines are for a layer from the surface up to $800 \mathrm{~m}$ $(\approx$ subcloud layer), and the purple lines for a layer from 800 to $1,600 \mathrm{~m}$ ( $\approx$ cloud layer). The $y$ axis corresponds to the percentage of flux carried by each wave number or eddy size. Because the horizontal grid size is $x \approx 250$ 

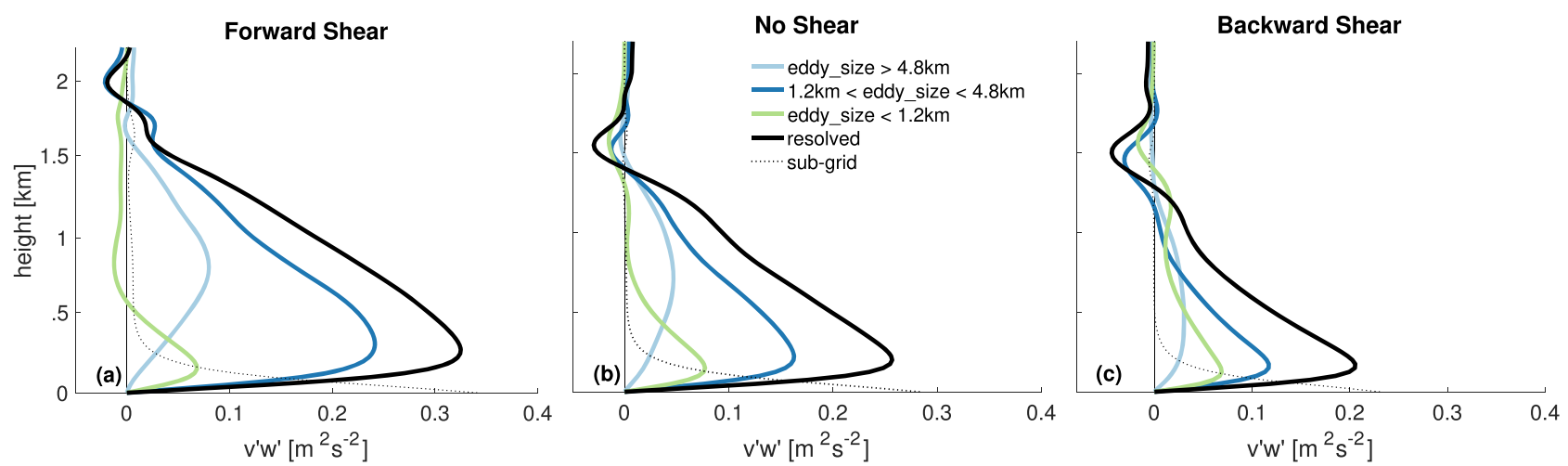

Figure 13. Meridional momentum fluxes divided by large, medium, and small scales in the last two hours of the simulation; (a) Forward shear, (b) no shear, (c) backward shear. The dotted lines are the subgrid flux as output from the LES. The black lines are the resolved flux.

$\mathrm{m}$, the smallest resolved eddy here is $500 \mathrm{~m}$, while the largest eddy that can be captured by the spectral analysis is $48 \mathrm{~km}$ (half the domain size). Above the surface (from $500 \mathrm{~m}$ on), all cospectra peak at eddy size $12 \mathrm{~km}$, which corresponds to about the size of the cold pools present in the simulations. A positive value implies a positive correlation of $v^{\prime}$ and $w^{\prime}$, while a negative value implies a negative correlation. A positive correlation means that the updrafts carry mostly slower than average winds (positive $v$ anomalies), while downdrafts mostly carry faster than average winds (negative $v$ anomalies). The normalized cospectra of the FW and NS cases are similar and always positive throughout both the subcloud and the cloud layer. In the subcloud layer, medium to small-scale eddies carry most of the flux, whereas in the cloud layer larger scales are important. In comparison, the BW shear case has more variable cospectra, which are on average positive in the subcloud layer, but negative in the cloud layer. This can be seen in Figure 13, where the momentum flux in the BW case is positive in the subcloud layer, and negative in the cloud layer (green line). The reason for this behavior can be found in the prescribed geostrophic and initial wind, Figure $5 \mathrm{~b}$. In the BW shear case, faster winds are transported upward, and deposited in the cloud layer (compare the initial profile and the mean state of the meridional wind in Figure 5b). This type of transport is described by a negative correlation of $v^{\prime}$ and $w^{\prime}$ in the cloud layer. Furthermore, the updrafts that are strong enough to perform this task mostly belong to the cloudy convective region, as can be seen in Figure 11i, where the faster winds $\left(v^{\prime}<0\right)$ in the cloud layer are found in the fourth quartile Q4. Of course, the negative $v^{\prime} w^{\prime}$ can also be generated by downdrafts that carry slower than average winds $\left(v^{\prime}>0\right)$. This is the case of downdrafts associated with local cloud circulation, and with cloud top entrainment. These processes are indeed responsible for the negative fluxes right above the boundary layer top also in the FW and the NS case (Figures $11 \mathrm{j}-111$ ). By analyzing the contributions to momentum flux given by updrafts and downdrafts, it emerges that along with downdrafts and cloud top entrainment, the updrafts are responsible for the negative flux only in the BW shear case (not shown here).

Looking at the spectral contributions accumulated over a range of sizes is perhaps more informative to answer whether small-scale turbulence or larger coherent mesoscale motions are more important. Here we divide the cospectra into three contributions: the large-, medium-, and small-scale eddies, which are separated by the dashed lines in Figure 12. The large scales range from half the domain size to $4.8 \mathrm{~km}$. The medium scales range from $4.8 \mathrm{~km}$ to $1.2 \mathrm{~km}$, and the remaining eddy sizes account for the small scales, which thus represent all turbulent and convective motions up to scales of about the boundary layer height. In this type of visualization, the area under the curves in Figure 12 is representative of the percentage of flux carried by a group of eddy sizes, rather than the individual percentage carried by each wave. In Figure 13 the momentum flux profiles that correspond to the cumulative cospectra of these three groups are displayed. The solid black lines correspond to the resolved momentum fluxes, and the thin dotted lines to the subgrid scale fluxes. The nature of the differences in flux profiles from the three eddy size groups is similar among the three simulations, but most pronounced in the FW shear case. Similar to what we have seen in the flux profiles for different CWV quartiles, different eddies carry different momentum flux profiles, and thus introduce a different tendency (deceleration or acceleration). The medium scales account for the largest part of the flux (50-70\%), and act as a friction. The small scales account for $\approx 30-40 \%$ of the flux in the subcloud layer and only for $10 \%$ in the cloud layer, and also introduce a friction. Small-scale eddies are therefore not 

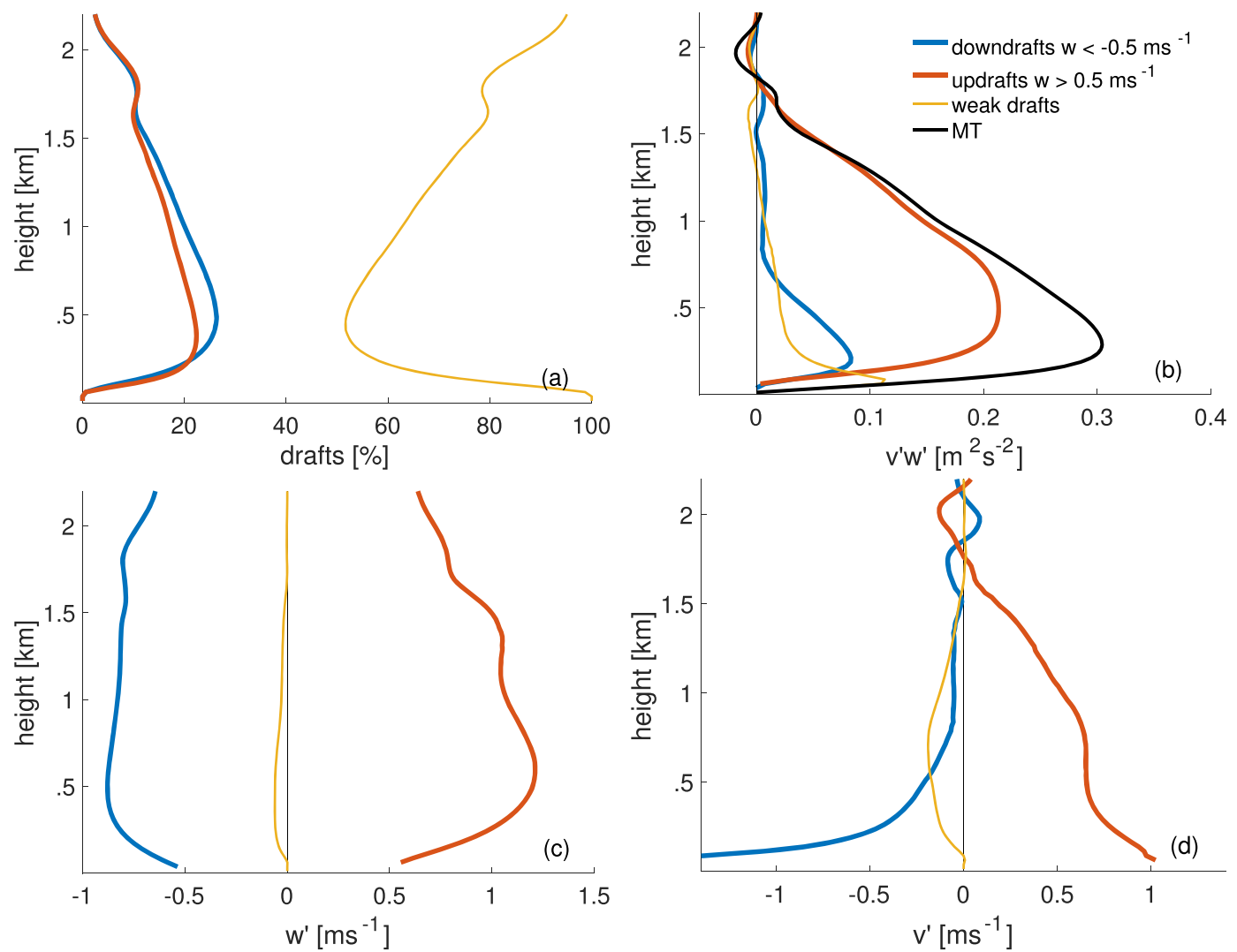

Figure 14. Decomposition of the FW shear case momentum flux at $14.5 \mathrm{hr}$ into strong downdrafts, strong updrafts, and weak drafts. (a) Relative fractions, (b) meridional momentum flux, (c) average vertical velocity perturbation, (d) average meridional velocity perturbation.

negligible, and as noted in Zhu (2015), the mass flux approach would miss to represent part of the flux contributed by these small scales.

Also not negligible, and perhaps equally important, are the larger scales, which contribute the most to the momentum transport in the second half of the subcloud layer (above $500 \mathrm{~m}$ ) and in the cloud layer, with a maximum below cloud base at $\approx 800 \mathrm{~m}$. In the cloud layer the large scales accounts for $\approx 40 \%$ of the momentum flux. What is best seen in the FW shear case, is that the flux of these larger scales increases with height in the subcloud layer, and only decreases above. This implies that such scales only introduce friction in the upper boundary layer (similar to what we saw for the moistest columns of the domain represented by Q4 in the previous section). In the subcloud layer, they lead to an acceleration of the meridional flow.

One reason for finding that the smallest scales are not more important in producing momentum flux than mesoscales, is that our simulations have a relatively coarse grid spacing at $250 \mathrm{~m}$, so that the smallest resolved eddy scale is already $500 \mathrm{~m}$. A finer resolution would increase the importance of smaller-scale turbulent motions. In another simulation where we refined the resolution by a factor of 2 , we find that this is especially true for cloud base, where the contribution by the small scales is doubled. In the subcloud layer the contribution of small scales will also be $10-30 \%$ larger.

\subsection{Momentum Transport Contribution by Updrafts and Downdrafts}

As seen in the previous sections, in the FW shear case the moist convective regions and the mesoscales would accelerate the upper part of the subcloud layer $(\approx 0.5-1 \mathrm{~km})$, effectively reducing the average friction given by vertical momentum mixing.

The natural question that arises is whether this local acceleration is due to the removal of slower than average winds from the subcloud layer, or from additional faster than average winds from above. In order to clarify this, we decompose the momentum flux of the FW shear case in strong updrafts $\left(w^{\prime}>0.5 \mathrm{~ms}^{-1}\right)$, strong downdrafts $\left(w^{\prime}<-0.5 \mathrm{~ms}^{-1}\right)$, and weak drafts $\left(-0.5 \leq w^{\prime} \leq 0.5 \mathrm{~ms}^{-1}\right)$. 

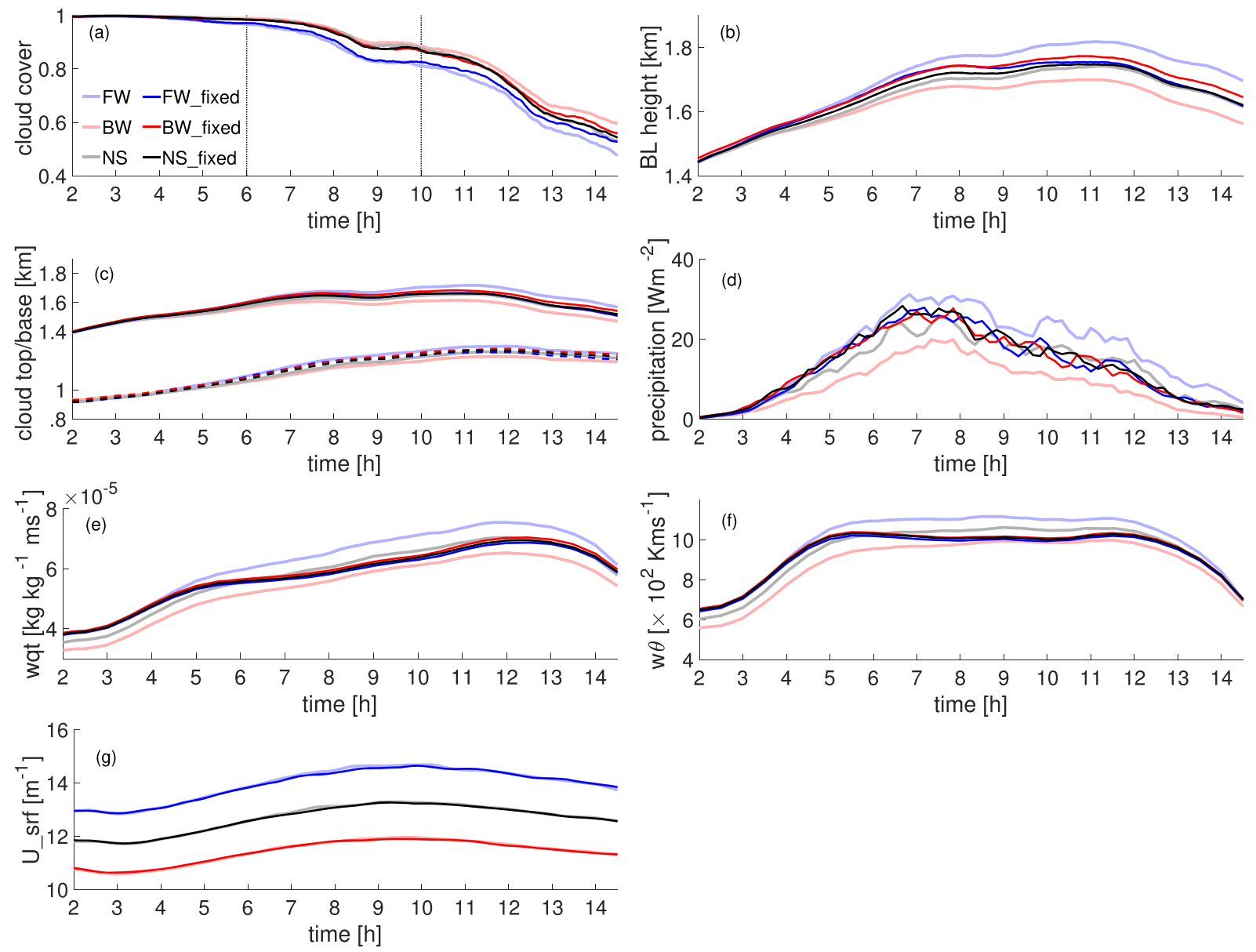

Figure 15. Time series of cloud cover (a), boundary layer height (b), mean cloud base (dashed lines) and cloud top (full lines) height (c), precipitation (d), surface fluxes of total water specific humidity (e), potential temperature (f), and surface wind speed (g). The shaded lines are the simulations with free surface winds, while the thin lines are the simulations with fixed surface winds.

With this sampling, strong updrafts and strong downdrafts represent $\approx 10-20 \%$ of the domain each (Figure 14a). The mean strong downdraft is constant in the whole layer $\left(\approx-0.8 \mathrm{~ms}^{-1}\right)$, suggesting that there exists some downward drafts starting from the cloud layer and reaching near the surface (Figure 14c). This can also be seen in $x-z$ snapshot visualizations, not shown here. However, the contribution of the strong downdrafts to the mean transport of momentum is bounded to the subcloud layer (Figure 14b), and is on average a friction. Hence, the downdrafts are not responsible for the accelerations given by convection and mesoscales, which are more likely a result of the upward motions, whereas slower winds are lifted from the subcloud layer and deposited in the cloud layer.

In conclusion, it is important to notice that the mean meridional wind perturbation in the strong downdrafts rapidly decreases in the subcloud layer and is very small $\left(\approx 0.1 \mathrm{~ms}^{-1}\right)$ in the cloud layer. This is not because there is little variance at these heights, but rather because the meridional winds caught in the downdrafts can vary substantially in the cloud layer, and the downdraft sampling does not capture a coherent structure.

\section{Sensitivity of CAO Evolution to Wind Shear}

In this section, we return to some of the first-order effects of wind shear on the boundary layer and cloud evolution. Wind shear is typically not considered as a cloud-controlling factor that plays a role in modulating the transition from stratocumulus to cumulus (De Roode et al., 2019). However, our results suggest that wind shear plays at least a secondary role in the evolution of the boundary layer and the cloud deck via its effects on surface winds and surface fluxes, as well as on turbulence and entrainment rates. 

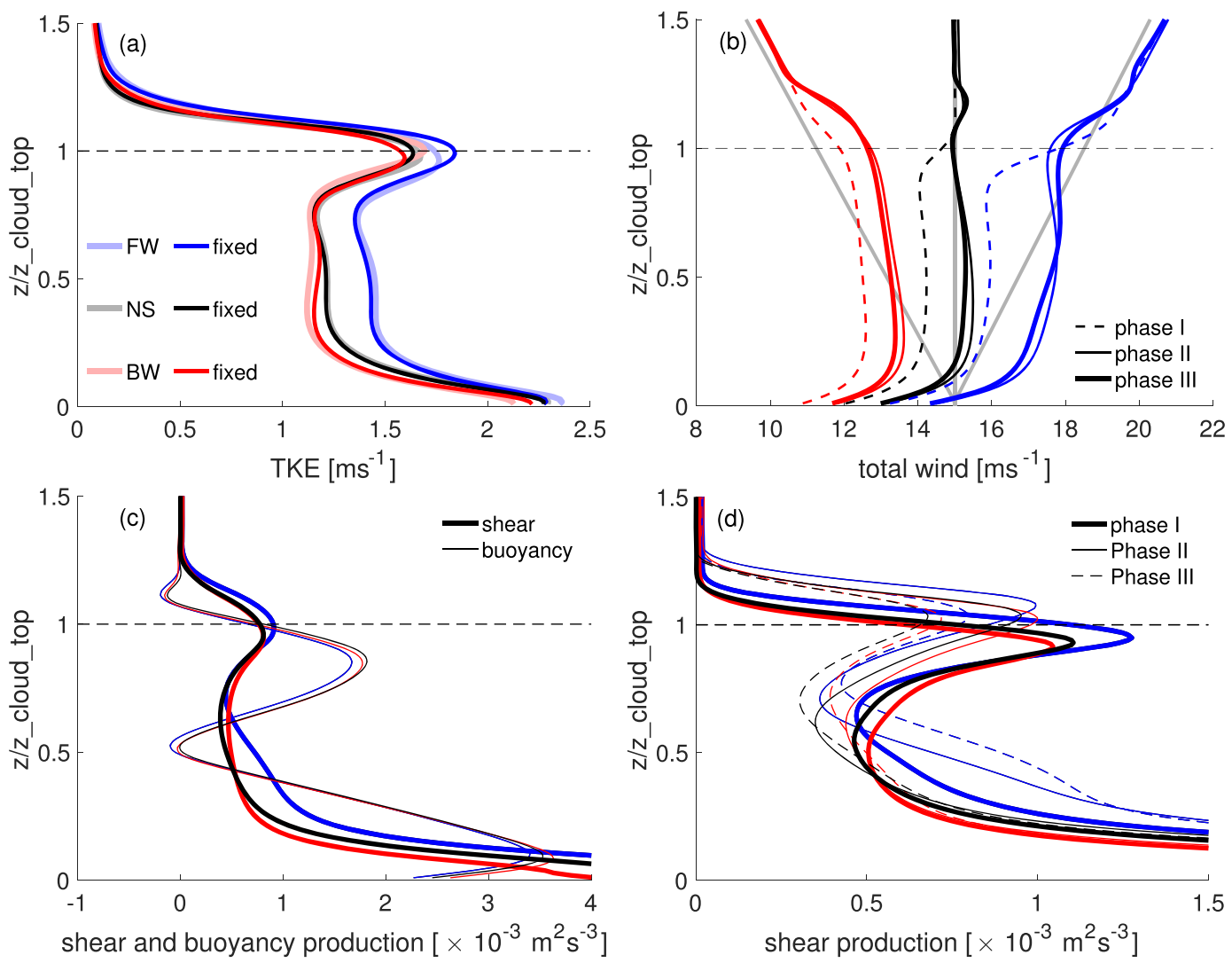

Figure 16. Mean TKE for simulations with interactive and fixed surface fluxes (a), total wind during the three phases (b), mean shear and buoyancy production terms of the TKE budget (c), and phases of the shear production term (d). Panels (b)-(d) are done for the fixed surface wind simulations. The gray lines are the meridional geostrophic (and initial) winds. The results refer to the resolved TKE budget.

As seen in the cloud fraction profiles (Figures $4 \mathrm{~d}-4 \mathrm{f}$ ), small but notable differences develop in the cloud transition as a function of the shear, which we show in more detail in Figure 15. The cloud cover of all three cases (Figure 15a) decreases with two distinctive jumps at hours 8 and 12. The boundary layer gradually deepens, as the cloud base and cloud top rise and precipitation is produced (Figures 15b-15d). The FW case develops a deeper boundary layer ( $\approx 100 \mathrm{~m}$ higher than the NS case and $\approx 200 \mathrm{~m}$ higher than the BW case), a slightly higher cloud top and more precipitation throughout the whole simulation. The reduction in cloud cover is also quicker in the FW shear case than in the NS and BW shear case. For example, the cloud cover is $80 \%$ after $9 \mathrm{hr}$, whereas in the other two cases the cloud cover drops to $80 \%$ only after $12 \mathrm{hr}$. At the end of the simulation, the FW shear case cloud cover is $\approx 50 \%$, while it is $\approx 60 \%$ in the NS case and $\approx 70 \%$ in the BW case.

The FW and BW shear case mix down faster and slower meridional winds, respectively, which leads to a few $\mathrm{ms}^{-1}$ difference in the surface wind speed. This results in enhanced surface fluxes of heat and moisture in the FW case (recall equation (1)). However, differences in the transition may also be explained by more direct effects of shear on entrainment mixing at the boundary layer top (Schulz \& Mellado, 2018). As found in Mellado et al. (2014) and Mellado (2017), a larger wind shear at the top of stratocumulus clouds can enhance the entrainment.

To disentangle direct effects of wind shear on turbulence and cloud from indirect effects of wind shear on the surface fluxes, modulated by momentum transport, we repeat our three experiments while keeping the surface winds fixed in the calculation of surface fluxes of heat and moisture. We take the surface wind speed of equation (1) as the time average of the NS case, which is $|\mathrm{U}|_{s}=12 \mathrm{~ms}^{-1}$. Because the thermodynamics are not strongly influenced by wind shear (Figures $5 \mathrm{c}$ and $5 \mathrm{~d}$ ), fixing the surface wind speed in the surface flux calculation is close to fixing the surface fluxes (Figures 15e and 5f). The surface momentum transport $u_{*}$ is still sensitive to surface wind speed as in equation (2), see Figure 15g. 
When the surface wind speed is fixed, the differences in boundary layer height, mean cloud base/top, and precipitation rate are largely removed (Figures 15a-15d, opaque lines). Still, between hours 6-12 the transition from stratocumulus to cumulus is more efficient under the FW shear. In the FW shear case, larger turbulence kinetic energy (TKE) is created both in the sub-cloud and in the cloud layer. Figures 16a and 16b show the mean TKE (for all cases, with both free (shaded lines) and fixed (thin dark lines) surface winds) and the mean total wind speed profiles for each phase (for interactive surface fluxes only), whereby the $y$ axis is normalized by the cloud top height.

Evidently, the TKE profiles depend more strongly on the type of shear than on the surface fluxes. Figure 16b shows that in the stratocumulus phases, the wind shear across the cloud top, and across the cloud layer, is larger in the FW case compared to the other cases. In fact, near cloud tops the wind shear in the FW case is twice as large as the BW and the NS case. This would support larger shear-driven turbulence (and TKE) in the FW case. The shear production term is indeed larger in the FW shear case in the subcloud layer, Figure 16c, and also at cloud top and in the cloud layer in the stratocumulus phase (phase I, Figure 16d).

\section{Discussion and Conclusions}

Motivated by a desire to better understand the importance of CMT in the momentum budget of midlatitude weather systems, we carried out simulations of a marine cold air outbreak (CAO), which we forced with varying baroclinicity (vertical wind shear).

Our first objective was to study how CMT influences the evolution of wind in a CAO system and whether it can explain weak low-level wind shear found in climatologies of CAOs. Indeed, as convection and clouds develop in the simulations wind speed profiles within the boundary layer become very well-mixed, with little vertical shear remaining. In comparison, zonal winds in BOMEX simulations still have considerable wind shear in the cloud layer (Brown, 1999). The difference may be explained by the different cloud fractions. In the stratocumulus transition case considered in this study, cloud fraction in the cloud layer approaches $100 \%$, whereas in BOMEX the fraction of cloudy updrafts is only $10 \%$.

Besides having little vertical wind shear in the boundary layer, our simulations also develop winds that are faster-than-geostrophic, such as in the cloud layer under backward shear (wind speed decreases with height) or in the subcloud layer under forward shear (wind speed increases with height). A quick explanation may be sought in vertical momentum transport, which can introduce relatively large momentum from near the surface into the cloud layer under backward shear, or relatively large momentum from the cloud layer into the subcloud layer under forward shear. However, our analysis of the kinetic energy budget shows that momentum transport always acts to slow down winds in the mean flow direction. The combination of momentum transport and a strong Coriolis force can increase wind speed beyond its geostrophic value. As winds are initially slowed down by surface drag and momentum transport, the wind turns across isobars into the direction of low pressure (which in our simulations is toward the east). Through the Coriolis force, this cross-isobaric or ageostrophic wind component acts as a reservoir of momentum for the mean meridional wind component, which is directed southward. The clockwise turning of ageostrophic wind in turn strengthens the southward flow, at least on the time scale of our simulations $(\approx 10 \mathrm{hr})$. On longer time scales, the ageostrophic wind reverses sign and winds gain a weaker southward component. In our simulations the overall wind turning is maybe exaggerated as we have ignored horizontal wind advection, which probably compensates for part of the wind turning introduced by the Coriolis force.

Although momentum transport always acts like a friction on the mean flow, the magnitude of the friction varies with height depending on the large-scale shear. Under forward shear, the friction introduced by momentum transport maximizes in the cloud layer. This is where the geostrophic wind forcing is the largest and the contrast with slow surface air pronounced. Under backward shear, momentum transport is largest in the subcloud layer, because the geostrophic wind forcing maximizes near cloud base (and as such, the shear between the surface and cloud base is large). The results exemplify how important the large-scale vertical shear is for explaining the nature of momentum transport.

Second, we wished to understand the relative influence of turbulent mixing versus coherent updrafts or mesoscale circulations (the convection) to the momentum flux. When we decompose the total momentum transport into contributions from areas that are strongly convecting (with large column water vapor, CWV) 
versus areas that experience mean subsidence and are dry (with low CWV), we find that momentum transport in the high CWV regions can accelerate subcloud layer flow in the mean direction (southward) under forward shear. This happens as (moist) convection transports momentum (with a weak southward component) out of the subcloud layer very efficiently. Accordingly, the momentum flux increases with height throughout the subcloud layer. The same result is found when decomposing the momentum flux spectrally and considering only eddy circulations on the scale of convection and mesoscales. Those mesoscale circulations help to accelerate the flow and oppose the friction introduced by smaller-scale eddies. Although the spectral analyses performed on the resolved fluxes underestimate the contribution by small-scale turbulence because our simulations have a relatively coarse horizontal resolution, they also hint at the compensating effects that are introduced by larger-scale mesoscale circulations, neither of which may be neglected when considering the effect of total momentum transport on large-scale winds.

Finally, we questioned how the cloud and boundary layer transitions that are so typical of CAOs depend on vertical shear through CMT. Via its influence on winds near the surface, momentum transport (and its interaction with the Coriolis force) impacts the surface fluxes and therefore the thermodynamic development of the boundary layer and the cloud transition. Under forward shear, larger surface wind speeds develop, which in turn lead to enhanced surface fluxes of heat and moisture and, thus, promote a deeper boundary layer, a higher cloud top, more precipitation and a faster transition to broken cumulus clouds (with a lower cloud cover). However, the differences are moderate, with a $\approx 10-20 \%$ increase in flux, $\approx 100$ m deeper boundary layer and 20-30\% lower cloud cover under forward shear. When excluding the surface wind speed response in the flux calculations, the forward shear still develops a faster transition. This implies that wind shear also has a direct effect on the transition. This happens through the production of more TKE and entrainment under forward shear, where the largest wind shear across the inversion is present, and is in line with previous work using DNS simulations of shear across the stratocumulus top (Mellado et al., 2014). Although this effect is much smaller than the influence of increasing SSTs across the transition, it exemplifies that large-scale wind shear is a factor that should be considered in studies on the sensitivity of stratocumulus to cumulus transitions, for instance in a changing climate.

Acknowledgments

We thank two reviewers for their comments on our manuscript. This project has received funding from the European Research Council (ERC) under the European Union's Horizon 2020 research and innovation program (Starting Grant Agreement 714918). The simulated data, along with the DALES code and the input files are stored in this site (https://doi.org/10. 4121/uuid:744df8d9-7232-46729110-f76bb25d69a0).

\section{References}

Boers, R., \& Melfi, S. H. (1987). Cold air outbreak during masex: Lidar observations and boundary-layer model test. Boundary-Layer Meteorology, 39(1-2), 41-51.

Boers, R., Melfi, S. H., \& Palm, S. P. (1991). Cold-air outbreak during gale: Lidar observations and modeling of boundary layer dynamics. Monthly Weather Review, 119(5), 1132-1150.

Bretherton, C. S., \& Blossey, P. N. (2017). Understanding mesoscale aggregation of shallow cumulus convection using large-eddy simulation. Journal of Advances in Modeling Earth Systems, 9, 2798-2821. https://doi.org/10.1002/2017MS000981

Brown, A. R. (1999). Large-eddy simulation and parametrization of the effects of shear on shallow cumulus convection. Boundary-Layer Meteorology, 91(1), 65-80.

Brümmer, B. (1996). Boundary-layer modification in wintertime cold-air outbreaks from the Arctic Sea ice. Boundary-Layer Meteorology, 80(1-2), 109-125.

Brümmer, B. (1997). Boundary layer mass, water, and heat budgets in wintertime cold-air outbreaks from the Arctic Sea ice. Monthly Weather Review, 125(8), 1824-1837.

Brümmer, B. (1999). Roll and cell convection in wintertime Arctic cold-air outbreaks. Journal of the Atmospheric Sciences, 56(15), 2613-2636.

Conzemius, R. J., \& Fedorovich, E. (2006). Dynamics of sheared convective boundary layer entrainment. Part I: Methodological background and large-eddy simulations. Journal of the Atmospheric Sciences, 63(4), 1151-1178.

De Roode, S. R., Frederikse, T., Siebesma, A. P., Ackerman, A. S., Chylik, J., Field, P. R., et al. (2019). Turbulent transport in the gray zone: A large eddy model intercomparison study of the constrain cold air outbreak case. Journal of Advances in Modeling Earth Systems, 11, 597-623. https://doi.org/10.1029/2018MS001443

Dee, D. P., Uppala, S. M., Simmons, A. J., Berrisford, P., Poli, P., Kobayashi, S., et al. (2011). The ERA-Interim reanalysis: Configuration and performance of the data assimilation system. Quarterly Journal of the Royal Meteorological Society, 137(656), 553-597.

Fedorovich, E., \& Conzemius, R. (2008). Effects of wind shear on the atmospheric convective boundary layer structure and evolution. Acta Geophysica, 56(1), 114-141.

Field, P. R., Brozková, R., Chen, M., Dudhia, J., Lac, C., Hara, T., et al. (2017). Exploring the convective grey zone with regional simulations of a cold air outbreak. Quarterly Journal of the Royal Meteorological Society, 143(707), 2537-2555.

Field, P. R., Cotton, R. J., McBeath, K., Lock, A. P., Webster, S., \& Allan, R. P. (2014). Improving a convection-permitting model simulation of a cold air outbreak. Quarterly Journal of the Royal Meteorological Society, 140(678), 124-138.

Fletcher, J., Mason, S., \& Jakob, C. (2016a). The climatology, meteorology, and boundary layer structure of marine cold air outbreaks in both hemispheres. Journal of Climate, 29(6), 1999-2014.

Fletcher, J. K., Mason, S., \& Jakob, C. (2016b). A climatology of clouds in marine cold air outbreaks in both hemispheres. Journal of Climate, 29(18), 6677-6692.

Fu, Q., \& Liou, K. N. (1992). On the correlated k-distribution method for radiative transfer in nonhomogeneous atmospheres. Journal of the Atmospheric Sciences, 49(22), 2139-2156.

Fu, Q., Liou, K. N., Cribb, M. C., Charlock, T. P., \& Grossman, A. (1997). Multiple scattering parameterization in thermal infrared radiative transfer. Journal of the Atmospheric Sciences, 54(24), 2799-2812. 
Grossman, R. L., \& Betts, A. K. (1990). Air-sea interaction during an extreme cold air outbreak from the eastern coast of the united states. Monthly Weather Review, 118(2), 324-342.

Heus, T., \& Jonker, H. J. (2008). Subsiding shells around shallow cumulus clouds. Journal of the Atmospheric Sciences, 65(3), $1003-1018$.

Heus, T., van Heerwaarden, C. C., Jonker, H. J. J., Siebesma, A. P., Axelsen, S., van den Dries, K., et al. (2010). Formulation of and numerical studies with the Dutch Atmospheric Large-Eddy Simulation (DALES). Geoscientific Model Development, 3, 415-444.

Kershaw, R., \& Gregory, D. (1997). Parametrization of momentum transport by convection. I: Theory and cloud modelling results. Quarterly Journal of the Royal Meteorological Society, 123(541), 1133-1151.

Kolstad, E. W. (2017). Higher ocean wind speeds during marine cold air outbreaks. Quarterly Journal of the Royal Meteorological Society, 143(706), 2084-2092.

Kolstad, E. W., Bracegirdle, T. J., \& Seierstad, I. A. (2009). Marine cold-air outbreaks in the North Atlantic: Temporal distribution and associations with large-scale atmospheric circulation. Climate Dynamics, 33(2-3), 187-197.

Larson, V. E., Domke, S., \& Griffin, B. M. (2019). Momentum transport in shallow cumulus clouds and its parameterization by higher-order closure. Journal of Advances in Modeling Earth Systems, 11, 3419-3442.

Marshall, J., \& Schott, F. (1999). Open-ocean convection: Observations, theory, and models. Reviews of Geophysics, 37(1), 1-64.

Mellado, J. P. (2017). Cloud-top entrainment in stratocumulus clouds. Annual Review of Fluid Mechanics, 49, 145-169.

Mellado, J. P., Stevens, B., \& Schmidt, H. (2014). Wind shear and buoyancy reversal at the top of stratocumulus. Journal of the Atmospheric Sciences, 71(3), 1040-1057.

Muller, C., \& Bony, S. (2015). What favors convective aggregation and why? Geophysical Research Letters, 42, 5626-5634. https://doi.org/ $10.1002 / 2015$ GL064260

Overland, J. E., \& Wilson, J. G. (1984). Mesoscale variability in marine winds at mid-latitude. Journal of Geophysical Research, 89(C6), $10,599-10,614$.

Papritz, L., Pfahl, S., Sodemann, H., \& Wernli, H. (2015). A climatology of cold air outbreaks and their impact on air-sea heat fluxes in the high-latitude South Pacific. Journal of Climate, 28(1), 342-364.

Papritz, L., \& Spengler, T. (2017). A Lagrangian climatology of wintertime cold air outbreaks in the Irminger and Nordic Seas and their role in shaping air-sea heat fluxes. Journal of Climate, 30(8), 2717-2737.

Pincus, R., \& Stevens, B. (2009). Monte Carlo spectral integration: A consistent approximation for radiative transfer in large eddy simulations. Journal of Advances in Modeling Earth Systems, 1, 1. https://doi.org/10.3894/JAMES.2009.1.1

Raasch, S. (1990). Numerical simulation of the development of the convective boundary layer during a cold air outbreak. Boundary-Layer Meteorology, 52(4), 349-375.

Renfrew, I. A., \& Moore, G. W. K. (1999). An extreme cold-air outbreak over the Labrador Sea: Roll vortices and air-sea interaction. Monthly Weather Review, 127(10), 2379-2394.

Sandu, I., Beljaars, A., Bechtold, P., Mauritsen, T., \& Balsamo, G. (2013). Why is it so difficult to represent stably stratified conditions in numerical weather prediction (NWP) models? Journal of Advances in Modeling Earth Systems, 5, 117-133. https://doi.org/10.1002/jame. 20013

Savic-Jovcic, V., \& Stevens, B. (2008). The structure and mesoscale organization of precipitating stratocumulus. Journal of the Atmospheric Sciences, 65(5), 1587-1605.

Schlemmer, L., Bechtold, P., Sandu, I., \& Ahlgrimm, M. (2016). Momentum transport in shallow convection. Reading, UK: European Centre for Medium-Range Weather Forecasts (ECMWF).

Schlemmer, L., Bechtold, P., Sandu, I., \& Ahlgrimm, M. (2017). Uncertainties related to the representation of momentum transport in shallow convection. Journal of Advances in Modeling Earth Systems, 9, 1269-1291. https://doi.org/10.1002/2017MS000915

Schulz, B., \& Mellado, J. P. (2018). Wind shear effects on radiatively and evaporatively driven stratocumulus tops. Journal of the Atmospheric Sciences, 75(9), 3245-3263.

Seifert, A., \& Beheng, K. D. (2001). A double-moment parameterization for simulating autoconversion, accretion and selfcollection. Atmospheric Research, 59, 265-281.

Seifert, A., \& Heus, T. (2013). Large-eddy simulation of organized precipitating trade wind cumulus clouds. Atmospheric Chemistry and Physics, 13(11), 5631-5645.

Siebesma, A. P., Bretherton, C. S., Brown, A., Chlond, A., Cuxart, J., Duynkerke, P. G., et al. (2003). A large eddy simulation intercomparison study of shallow cumulus convection. Journal of the Atmospheric Sciences, 60(10), 1201-1219.

Tomassini, L., Field, P. R., Honnert, R., Malardel, S., McTaggart-Cowan, R., Saitou, K., et al. (2017). The "grey zone" cold air outbreak global model intercomparison: A cross evaluation using large-eddy simulations. Journal of Advances in Modeling Earth Systems, 9, 39-64 https://doi.org/10.1002/2016MS000822

Wang, H., \& Feingold, G. (2009). Modeling mesoscale cellular structures and drizzle in marine stratocumulus. Part I: Impact of drizzle on the formation and evolution of open cells. Journal of the Atmospheric Sciences, 66(11), 3237-3256.

Wayland, R. J., \& Raman, S. (1989). Mean and turbulent structure of a baroclinic marine boundary layer during the 28 January 1986 cold-air outbreak (GALE 86). Boundary-Layer Meteorology, 48(3), 227-254.

Weisman, M. L., \& Klemp, J. B. (1984). The structure and classification of numerically simulated convective storms in directionally varying wind shears. Monthly Weather Review, 112(12), 2479-2498.

Zhang, Y., Stevens, B., \& Ghil, M. (2005). On the diurnal cycle and susceptibility to aerosol concentration in a stratocumulus-topped mixed layer. Quarterly Journal of the Royal Meteorological Society, 131(608), 1567-1583.

Zhu, P. (2015). On the mass-flux representation of vertical transport in moist convection. Journal of the Atmospheric Sciences, 72(12), $4445-4468$.

Zolina, O., \& Gulev, S. K. (2003). Synoptic variability of ocean-atmosphere turbulent fluxes associated with atmospheric cyclones. Journal of Climate, 16(16), 2717-2734. 